\title{
Emergent strings, duality and weak coupling limits for two-form fields
}

\author{
Seung-Joo Lee, ${ }^{a}$ Wolfgang Lerche ${ }^{a}$ and Timo Weigand ${ }^{a, b}$ \\ ${ }^{a}$ CERN, Theory Department, \\ 1 Esplande des Particules, Geneva 23, CH-1211, Switzerland \\ ${ }^{b}$ PRISMA Cluster of Excellence and Mainz Institute for Theoretical Physics, \\ Johannes Gutenberg-Universität, \\ Staudingerweg 9, 55099 Mainz, Germany \\ E-mail: seung.joo.lee@cern.ch, wolfgang.lerche@cern.ch, \\ timo.weigand@cern.ch
}

ABSTRACT: We systematically analyse weak coupling limits for 2-form tensor fields in the presence of gravity. Such limits are significant for testing various versions of the Weak Gravity and Swampland Distance Conjectures, and more broadly, the phenomenon of emergence. The weak coupling limits for 2-forms correspond to certain infinite-distance limits in the moduli space of string compactifications, where asymptotically tensionless, solitonic strings arise. These strings are identified as weakly coupled fundamental strings in a dual frame, which makes the idea of emergence manifest. Concretely we first consider weakly coupled tensor fields in six-dimensional compactifications of F-theory, where the arising tensionless strings play the role of dual weakly coupled heterotic strings. As the main part of this work, we consider certain infinite distance limits of Type IIB strings on K3 surfaces, for which we show that the asymptotically tensionless strings describe dual fundamental Type IIB strings, again on K3 surfaces. By contrast the analogous weak coupling limits of M-theory compactifications are found to correspond to an F-theory limit where an extra dimension emerges rather than tensionless strings. We comment on extensions of our findings to four-dimensional compactifications.

KeYwords: String Duality, Superstrings and Heterotic Strings

ArXiv EPrint: 1904.06344 


\section{Contents}

1 Introduction and summary 1

2 Emergent heterotic string from 6d F-theory 4

2.1 Geometric setup 5

2.2 Weak coupling limit for 2-form potentials 7

$\begin{array}{lll}2.3 & \text { Emergent heterotic string at weak coupling } & 10\end{array}$

$3 \quad$ Emergent fundamental strings from Type IIB strings on K3 11

3.1 Large distance limits in the moduli space $\mathcal{M}_{(5,21)} \quad 12$

3.2 Geometric weak coupling limits in Kähler moduli space of K3 15

$\begin{array}{ll}\text { 3.3 The solitonic string as a Type IIB fundamental string } & 19\end{array}$

3.4 Emergent strings and duality in the geometric weak coupling limit 20

4 Emergent F-theory from M-theory on K3 in weak coupling limit 23

5 Conclusions, and prospects for four-dimensional strings 25

\section{Introduction and summary}

Within the web of recent Quantum Gravity Conjectures, reviewed in [1, 2], the Swampland Distance Conjecture [3] plays a central role. It postulates that as one approaches a point at infinite distance in the moduli space of any theory of quantum gravity, a tower of infinitely many states becomes asymptotically massless. The resulting breakdown of the original effective theory explains microscopically why the critical point is located at infinite distance in moduli space. In the context of a gauge theory, the infinite tower of states becoming light in the weak coupling limit includes states that satisfy the Weak Gravity Conjecture [4], thus linking the two conjectures quite directly $[5-7]$ (see also $[8,9]$ ). This has been confirmed by detailed investigations of the towers of massless states appearing at infinite distance in string theory [10-18]. The arguments do in general not rely on BPS properties of the particles $[11,12,15]$ and hence even apply to theories with $N=1$ supersymmetry in four dimensions, as analyzed in [15, 19]. Refined versions of the Swampland Distance Conjecture [7, 20], examined further in string theory in [21], have potentially important consequences for cosmological models relying on super-Planckian excursions of scalar fields. Emergent towers of states have been proposed in [22] as a rationale behind the recent de Sitter conjectures [23] at weak coupling. An extensive account of the role of such towers of states, along with a survey of the recent literature, can be found in [2].

A celebrated result in string theory [24] is the logarithmic divergence of gauge couplings in four-dimensional string compactifications, which arises from a finite number of 
states becoming massless at special finite distance points in moduli space. By contrast, in a situation at infinite distance, an infinite number of states is supposedly becoming asymptotically massless. As a result the logarithmic divergence can turn into a polynomial one. This phenomenon is tied to the idea of emergence $[2,10,25-27]$ : the fields whose inverse couplings diverge at the infinite distance point are understood as a collective phenomenon associated with the appearance of a tower of massless states. Specifically, for a gauge field that becomes weakly coupled at infinite distance, one may in principle successively integrate in the infinite tower of states as one passes to higher and higher energy scales. The effect is to make the gauge field eventually strongly coupled and disappear as a fundamental field at high energies [2]. Such behaviour by itself is not limited to four dimensions.

What has been studied in great detail in the literature up to this point is the infinite distance regime in moduli space near the weak coupling limit of some gauge field, which is a 1-form potential. The resulting nearly massless tower of states a priori refers to particles that are charged under this gauge field and contribute to the renormalization of the gauge coupling. This manifestation of emergence is tied to a gauge subsector and the respective charged particles.

In this note we widen the scope of emergence and systematically analyze the weak coupling regime for 2-form gauge potentials. The most natural setting for such theories is in six dimensions, which is the focus of this work. ${ }^{1}$ Since 2 -forms couple to strings, the tower of states associated with them are not just certain charged fields, but the whole string excitation spectrum. Within the framework of string theory, six-dimensional theories with 2 -forms can be constructed by compactifying Type IIB strings on complex 2-folds, denoted by $B_{2}$. If $B_{2}$ is a $\mathrm{K} 3$ surface, the theory preserves 16 supercharges and corresponds to an effective $N=(2,0)$ supergravity theory. On suitably curved Kähler surfaces, extra 7 -branes are needed for consistency and supersymmetry is broken to $N=(1,0)$. Models of the latter type fall into the framework of F-theory [49-51]. By duality, the latter type of theories may also be described in terms of heterotic strings, but our starting point will be the Type IIB/F-Theory perspective.

One of our main results is that for such six-dimensional Type IIB or F-Theory theories, any weak coupling limit of some 2-form gauge potential leads to a tensionless solitonic string which couples precisely to that weakly coupled 2-form. As we will see, this string corresponds to a weakly coupled, critical fundamental string. The weak coupling limit of the 2-form hence forces, in a sense, the change to a new duality frame, in terms of which most excitations of the original perturbative string decouple from the low energy spectrum, while the excitations of the new fundamental string become light. The degenerations required to take the weak coupling limit occur on the boundary of the Kähler moduli space. $^{2}$ As we will show, the weak coupling limit of the 2-form potential inevitably requires the shrinking of a curve of vanishing self-intersection. The asymptotically tensionless string then arises as a soliton obtained by wrapping a D3-brane along this shrinking cycle.

\footnotetext{
${ }^{1}$ In four dimensions, 2-forms are dual to axionic fields, and Quantum Gravity Conjectures such as generalizations of the Weak Gravity Conjecture and others may have important implications e.g. for the structure of instanton corrections [20, 21, 28-48]. The recent [17] studies such instanton corrections in the context of emergence and the Swampland Distance Conjecture.

${ }^{2}$ For K3 this is true as long as we focus on attractive K3s as will be explained in section 3.
} 
As mentioned above, the objects which become mass- or tensionless in such weak coupling limits are not just strings per se, but these come together with the entire tower of their particle excitations (as viewed from the dual, perturbative frame). The only other potential source of nearly massless states are Kaluza-Klein excitations of these string states, which may arise if in the infinite distance limit some submanifold of the compactification space simultaneously becomes large. We will show that this in fact happens generically; the KaluzaKlein scale of these states turns out to be linked to the new fundamental string scale, but it is relatively suppressed by the volume $\mathcal{V}$ of the internal space $B_{2}$. To the extent that the volume is kept fixed so as to keep gravity dynamical, both towers of states become massless in the same large distance limit. Thus for fixed $\mathcal{V}$, there is no regime where a Kaluza-Klein tower is the only tower becoming light, and in this sense no new dimensions open up in the effective field theory. The appearance of a tensionless string is crucial for this behaviour.

Our observations appear interesting also from the perspective of the purported phenomenon of emergence: the computational challenge behind providing evidence for this proposal is to show how the tower of light states reproduces the observed singularities in the couplings of the effective theory at infinite distance. This has been demonstrated qualitatively in various explicit string realizations [10, 11, 13, 14, 26, 27], without detailed knowledge of the contributions of the nearly massless states at each mass level. On the other hand, for the types of large distance limits that we will discuss in this note, emergence is manifest: the perturbative effective theory at large distance is by definition the result of integrating out the full tower of fundamental string excitations and of Kaluza-Klein excitations. In the weak coupling limit, non-perturbative effects play no role. The states which become light are precisely the fundamental string excitations plus their Kaluza-Klein tower in the new duality frame - be it the dual heterotic or the dual Type IIB frame. Integrating out these states is guaranteed, by construction, to exactly reproduce the effective supergravity couplings.

To appreciate the role of the emergent strings, it is fruitful to compare the situation for Type IIB/F-theory to the corresponding situation in Type IIA/M-theory. The analogous question to study is the weak coupling limit for 1-form gauge potentials of M-theory on K3. The limit is again controlled by the Kähler parameters, at least as long as we focus on attractive K3s. In such cases the weak coupling limit will be found to be identical to taking the F-theory limit. More specifically, the tower of asymptotically massless states are now particles (with no strings attached), which arise from M2-branes that wrap a shrinking curve. As we will show, the shrinking curve corresponds to a genus-one fiber within the attractive K3. This is a consequence of the geometry of the weak coupling limit even without further assumptions on the details of an elliptic fibration structure. ${ }^{3}$ Interpreting these states as Kaluza-Klein states of a dual F-theory on $S^{1}$, one encounters an extra dimension unfolding in the weak coupling limit. Interestingly, the unfolding of an extra dimension has also been argued in [52] to be a consequence of a strong version of the scalar gravity conjecture [53]. By contrast, for the Type IIB version of the Kähler

\footnotetext{
${ }^{3}$ The F-theory limit of shrinking fiber volume for elliptically fibered Calabi-Yau 3-folds has been studied in [14] as a special case within a more general analysis of infinite distance singularities in Kähler moduli space.
} 
moduli degeneration we consider here, where certain 2-forms become weakly coupled in the presence of gravity and tensionless strings emerge, the large distance limit is physically very different and, in particular, no extra dimension opens up, in the sense described above.

In summary, the main results of our analysis of weak coupling limits of 2-forms are as follows:

- In section 2 we will show that in $N=(1,0)$ supersymmetric F-theory compactifications to six dimensions, tensionless heterotic strings emerge in the weak coupling limit of 2 -forms. This is closely related to our previous work [11, 12] which was aimed at weakly coupled gauge fields.

- The main body of our work is in section 3, where we consider six-dimensional compactifications of Type IIB strings with $N=(2,0)$ supersymmetry. Out of the five possible large distance limits (two of which are trivial), we will focus on one particular of such limits, and find that the tensionless string that arises is again dual to the Type IIB string, probing some elliptically fibered K3 surface. Thus, in some sense, the theory reproduces itself at large distance, upon a change of duality frame. This yields a six-dimensional analog of the famous picture of the moduli space of 10dimensional M-theory, where all large distance limits correspond to emerging weakly coupled strings (except, of course, for 11-dimensional supergravity). This is consistent with the intuition that a gravitational theory with a weakly coupled 2 -form must be a theory of critical, fundamental strings.

- In section 4 we contrast the previous analysis with M-Theory compactifications to seven dimensions. Here we see that in the respective large distance limits, towers of asymptotically massless particles arise, which are associated with an emerging extra dimension rather than with a tensionless string in the same number of dimensions.

- A similar picture of an emerging fundamental string governs at least some of the weak coupling limits also for F-theory/Type IIB compactifications to four dimensions, as we briefly sketch in section 5 .

Note added. While this work was readied for publication, we received [17] and [18], which partially overlap with our results in that they study aspects of large distance limits in relation to tensionless strings and instantons in four-dimensional string compactifications.

\section{Emergent heterotic string from 6d F-theory}

We begin by analyzing weak coupling limits for 2 -form potentials in general six-dimensional F-theory compactifications with $N=(1,0)$ supersymmetry. The main result of this section can be summarized as follows:

Claim 1 Consider a limit where some 2-form gauge potential in F-theory compactified to six dimensions becomes asymptotically weakly coupled, while gravity is kept dynamical. This limit is at infinite distance in Kähler moduli space, and there emerges an asymptotically 
tensionless heterotic string, which is charged under the weakly coupled 2-form potential in question. The weak coupling limit corresponds to a change of duality frame to the one of a perturbative heterotic string.

The relevant limit in Kähler moduli space will be found to be identical to the weak coupling limits for 7-brane gauge theories in F-theory studied already in $[11,12]$. The new aspects of the discussion in this section refer to the relation of this degeneration limit to the weak coupling regime of 2 -form fields. It furthermore paves the way for an understanding of the weak coupling limits of Type IIB strings on K3 surfaces, which will be studied in section 3 .

\subsection{Geometric setup}

Consider compactifications of F-theory to six dimensions [49-51]. The compactification space is given by a Calabi-Yau threefold, which is an elliptic fibration over some Kähler surface $B_{2}$ with non-trivial anti-canonical bundle, $\bar{K}_{B_{2}} \cdot{ }^{4}$ The low-energy effective theory is described by a six-dimensional, chiral $N=(1,0)$ supergravity theory. The various 2 -form gauge potentials result from the dimensional reduction of the Type IIB Ramond-Ramond 4-form, $C_{4}$, with respect to some basis of harmonic $(1,1)$ forms on $B_{2}$ :

$$
C_{4}=B^{\alpha} \wedge \omega_{\alpha}, \quad \omega_{\alpha} \in H^{1,1}\left(B_{2}\right) .
$$

The dual basis $\left\{\omega^{\alpha}\right\}$ of curve classes on $B_{2}$, defined via $\omega^{\alpha} \cdot \omega_{\beta}=\delta_{\beta}^{\alpha}$, is related to $\left\{\omega_{\alpha}\right\}$ as

$$
\omega^{\alpha}=\Omega^{\alpha \beta} \omega_{\beta} .
$$

Here $\Omega^{\alpha \beta}=\omega^{\alpha} \cdot \omega^{\beta}$ is the inverse of the intersection form

$$
\Omega_{\alpha \beta}=\omega_{\alpha} \cdot \omega_{\beta} \equiv \int_{B_{2}} \omega_{\alpha} \wedge \omega_{\beta} .
$$

The volume of $B_{2}$ is computed in terms of the Kähler form

$$
J=j^{\alpha} \omega_{\alpha}
$$

as

$$
\mathcal{V}=\frac{1}{2} J \cdot J
$$

and throughout this article we keep $\mathcal{V}$ at a fixed value to ensure that gravity remains dynamical. The kinetic terms for the collection of 2 -form fields $B^{\alpha}$,

$$
S_{\text {kin }}=\int_{\mathbb{R}^{1,5}} \frac{M_{\mathrm{Pl}}^{4}}{2} \sqrt{-g} R-\frac{2 \pi}{4} \int_{\mathbb{R}^{1,5}} g_{\alpha \beta} d B^{\alpha} \wedge * d B^{\beta}+\ldots \quad \text { with } \quad M_{\mathrm{Pl}}^{4}=4 \pi \mathcal{V},
$$

are then controlled by the following coupling matrix:

$$
g_{\alpha \beta}=\int_{B_{2}} \omega_{\alpha} \wedge * \omega_{\beta}=\frac{j_{\alpha} j_{\beta}}{\mathcal{V}}-\Omega_{\alpha \beta} .
$$

\footnotetext{
${ }^{4}$ For an introduction to some of the techniques used to describe such backgrounds, we refer e.g., to [54, 55] and references therein.
} 
The pseudo-action (2.6) follows by dimensional reduction from the 10d action in the Einstein frame with $\ell_{s} \equiv 1$. Note that (2.7) is an immediate consequence of the fact that on a Kähler surface of volume $\mathcal{V}$

$$
* \omega_{\alpha}=\frac{J \cdot \omega_{\alpha}}{\mathcal{V}} J-\omega_{\alpha}
$$

As usual for six-dimensional $N=(1,0)$ supergravity, the gauge invariant field strengths, $H^{\alpha}$, derived from the 2 -form potentials $B^{\alpha}$, are subject to the self-duality condition

$$
g_{\alpha \beta} * H^{\beta}=\Omega_{\alpha \beta} H^{\beta},
$$

where the field strengths involve in addition suitable Chern-Simons terms which are responsible for the correct cancellation of local anomalies. Details can be found e.g. in [56] and in the references therein.

The 2-form gauge potentials couple to effective strings in $\mathbb{R}^{1,5}$ which arise from D3branes which wrap holomorphic curves on $B_{2}$. To discuss this, it is useful to introduce another basis of curve classes

$$
C^{I}=C_{\alpha}^{I} \omega^{\alpha}
$$

and their dual divisors $C_{J}=C_{J}^{\alpha} \omega_{\alpha}$ such that $C^{I} \cdot C_{J}=\delta_{J}^{I}$. We will also need the intersection form

$$
\Omega_{I J}=C_{I} \cdot C_{J}=C_{I}^{\alpha} C_{J}^{\beta} \Omega_{\alpha \beta},
$$

as well its inverse, $\Omega^{I J}$, in order to lower and raise the $I$ index.

In terms of these, the coupling of the 2-forms to the string obtained by wrapping a D3-brane along the curve $C^{I}$ derives from the D3-brane Chern-Simons couplings as follows:

$$
\begin{aligned}
S_{\mathrm{CS}} & =2 \pi \int_{\mathbb{R}^{1,1} \times C^{I}} C_{4}=2 \pi \int_{\mathbb{R}^{1,1} \times B_{2}}\left(B^{\alpha} \wedge \omega_{\alpha}\right) \wedge\left(C_{\beta}^{I} \omega^{\beta}\right) \\
& =: 2 \pi \int_{\mathbb{R}^{1,1}} B^{I} .
\end{aligned}
$$

Here, the 2 -forms $B^{I}$ are related to the fields $B^{\alpha}$ as

$$
B^{I}=C_{\alpha}^{I} B^{\alpha} .
$$

The matrix $g_{I J}$ of kinetic terms associated with these linear combinations $B^{I}$ is then obtained by equating

$$
S_{\mathrm{kin}}=-\frac{2 \pi}{4} \int_{\mathbb{R}^{1,5}} g_{\alpha \beta} d B^{\alpha} \wedge * d B^{\beta}=-\frac{2 \pi}{4} \int_{\mathbb{R}^{1,5}} g_{I J} d B^{I} \wedge * d B^{J},
$$

which yields

$$
g_{I J}=C_{I}^{\alpha} C_{J}^{\beta} g_{\alpha \beta}=\frac{1}{\mathcal{V}}\left(J \cdot C_{I}\right)\left(J \cdot C_{J}\right)-C_{I} \cdot C_{J} .
$$

As long as the divisors $C_{I}$ are effective, the matrix $g_{I J}$ depends on the volumes of $C_{I}$ via

$$
\operatorname{vol}\left(C_{I}\right)=J \cdot C_{I}
$$

Note however that the expression (2.16) holds also for more general divisor classes, irrespective of whether they admit a holomorphic representative. 


\subsection{Weak coupling limit for 2-form potentials}

With this preparation we can now analyse the possible weak coupling limits for the 2 -form potentials, $B^{I}$, under the side condition that gravity is not decoupled. On general grounds, a tensor field can only be weakly coupled if it is a linear combination of a self-dual and antiself-dual tensor field. Sten-dimensionalix-dimensional $N=(1,0)$ supergravity contains one self-dual tensor, which is part of the gravity multiplet, along with $n_{T}$ anti-self-dual tensors in tensor multiplets. A weak coupling limit must thus single out some linear combination of anti-self-dual tensors to combine with the gravitensor to form the desired weakly coupled tensor field, T. In a suitable basis, its kinetic terms can be written as

$$
S_{\text {kin }}=\int_{\mathbb{R}^{1,5}} \frac{M_{\mathrm{Pl}}^{4}}{2} \sqrt{-|g|} R-\frac{2 \pi}{4}\left(S^{2} d \mathrm{~T} \wedge * d \mathrm{~T}+S^{-2} d \tilde{\mathrm{T}} \wedge * d \tilde{\mathrm{T}}\right)+\ldots \quad \text { for } \quad S \rightarrow \infty .
$$

Here $S$ can be interpreted as a "dilaton" that couples to the weakly coupled tensor field T and determines its inverse gauge coupling. On the other hand, the dual tensor $\tilde{\mathrm{T}}$, defined by $^{5}$

$$
d(\mathrm{~T}+\ldots)=S^{-2} * d(\tilde{\mathrm{T}}+\ldots),
$$

becomes strongly coupled in the limit $S \rightarrow \infty$. Note that in this limit, the remaining anti-self-dual tensors do not mix with $\mathrm{T}$ nor with $\tilde{\mathrm{T}}$.

To identify such a limit for the system of tensor fields in (2.15), we analyze the matrix of gauge kinetic terms, $g_{I J}$, for the 2 -form gauge fields, $B^{I}$. In order for a weak coupling limit for (at least) one linear combination of potentials to be attained, (at least) one eigenvalue of $g_{I J}$ must tend to infinity. At the same time, we must keep the volume of the base, $B_{2}$, fixed as in (2.5), in order to keep gravity dynamical. This means that there must exist at least one divisor class, $C$, for which

$$
J \cdot C \rightarrow \infty, \quad \text { with } \quad \frac{1}{2} J \cdot J=\mathcal{V} \quad \text { fixed } .
$$

This type of geometric degeneration limit coincides precisely with the type of infinite distance limits that were analyzed in full generality in refs. [11, 12]. The motivation of $[11,12]$ to study this limit was a priori independent of the goal of the present paper: these references consider the weak coupling limit for 1 -form gauge fields localised on 7 -branes that wrap certain divisors, $C$, of $B_{2}$. The inverse coupling of these gauge theories is proportional to $J \cdot C$, and therefore (2.20) governs their weak coupling limit as well. We thus see that the same geometric limit also governs the weak coupling regime of the 2 -form potentials, whose inverse coupling matrix is given by (2.16).

It was shown in $[11,12]$ that whenever a limit of the form $(2.20)$ can be taken, the Kähler form must behave asymptotically as

$$
J=t J_{0}+\sum_{i} \frac{a_{i}}{2 t} J_{i} \quad \text { for } \quad t \rightarrow \infty
$$

\footnotetext{
${ }^{5}$ The dots stand for the Chern-Simons corrections to the field strength, which play no role for us. They will be omitted in the sequel.
} 
where $J_{0}$ and $J_{i}$ are generators of the Kähler cone and $C \cdot J_{0} \neq 0$. Here $J_{0}$ is singled out as the generator of the Kähler cone whose coefficient scales to infinity, which is thus responsible for the volume of some curve class to diverge. As proven in [11], eq. (2.21) is the most general ansatz that realises the limit (2.20). In order for the base volume to remain finite in the limit $t \rightarrow \infty$, as in (2.5), the Kähler generators $J_{0}$ and $J_{i}$ must satisfy

$$
J_{0} \cdot J_{0}=0, \quad \sum_{i} \frac{a_{i}}{2} J_{i} \cdot J_{0}=\mathcal{V}+\mathcal{O}\left(1 / t^{2}\right),
$$

where $a_{i}$ are parameters that stay finite in the limit $t \rightarrow \infty$.

An important property of the asymptotic behavior (2.21) is that the class associated with $J_{0}$ necessarily contains a holomorphic, rational curve

$$
C^{0}:=J_{0} \quad \text { with } \quad C^{0} \cdot C^{0}=0,
$$

whose volume vanishes in the limit as

$$
\operatorname{vol}\left(C^{0}\right)=\sum_{i} \frac{a_{i}}{2 t} J_{i} \cdot J_{0}=\frac{\mathcal{V}}{t}+\mathcal{O}\left(1 / t^{3}\right) \quad \text { for } \quad t \rightarrow \infty .
$$

This implies that a D3-brane wrapping this curve $C^{0}$ gives rise to a solitonic string in $\mathbb{R}^{1,5}$ with tension

$$
T_{C^{0}}=2 \pi \operatorname{vol}\left(C^{0}\right)=2 \pi \frac{\mathcal{V}}{t}+\mathcal{O}\left(1 / t^{3}\right)
$$

in the frame defined via (2.6).

Before giving an interpretation of this solitonic string in section 2.3, we now show that it becomes weakly coupled in the tensionless limit. Viewing the string as the object charged under some given, definite linear combination of 2 -forms, it becomes weakly coupled whenever the diagonal kinetic term (2.16) of the relevant 2-form diverges and there is no significant kinetic mixing involving this 2 -form. To check this explicitly, consider the following basis of curve classes

$$
\left\{C^{I}\right\}=\left\{C^{0}:=J_{0}, \quad C^{i}:=J_{i}\right\} .
$$

Note that we are only using the property that $C^{0}$ contains a holomorphic curve class, as established in [11], while the remaining classes, $C^{i}$, need not have this property. We are interested in the kinetic terms for the linear combination $B^{0}$ of 2 -forms that couples to $C^{0}$ as shown in (2.12). In particular, we need to analyze the limit $t \rightarrow \infty$ of the kinetic terms $g_{00}$ and $g_{0 i}$, where $g_{I J}$ is defined in (2.16).

To this end, introduce the following dual basis of divisors $C_{I}$ which has the properties

$$
C_{0} \cdot C^{0}=1, \quad C_{i} \cdot C^{0}=0, \quad C_{0} \cdot C^{i}=0, \quad C_{i} \cdot C^{j}=\delta_{i}^{j} .
$$

In the limit (2.21),

$$
\begin{aligned}
& J \cdot C_{0}=t J_{0} \cdot C_{0}+\sum_{i} \frac{a_{i}}{2 t} J_{i} \cdot C_{0}=t, \\
& J \cdot C_{i}=t J_{0} \cdot C_{i}+\sum_{j} \frac{a_{j}}{2 t} J_{j} \cdot C_{i}=\frac{a_{i}}{2 t},
\end{aligned}
$$


where we used (2.26). As a result, the metric (2.16) becomes

$$
\begin{array}{ll}
g_{00} & =\frac{t^{2}}{\mathcal{V}}-C_{0} \cdot C_{0}, \quad g_{0 i}=\frac{a_{i}}{2 \mathcal{V}}-C_{0} \cdot C_{i} \\
g_{i j} & =\frac{a_{i} a_{j}}{4 \mathcal{V} t^{2}}-C_{i} \cdot C_{j} .
\end{array}
$$

In the limit $t \rightarrow \infty$, the mixing of the 2 -form $B^{0}$ with the remaining 2 -forms becomes negligible in the sense that $g_{0 i} / g_{00} \rightarrow 0$. Together with the fact that $g_{00} \sim t^{2} \rightarrow \infty$, this guarantees that the string associated with $C^{0}$ becomes weakly coupled. Importantly, the remaining kinetic couplings, $g_{i j}$, stay finite in the limit $t \rightarrow \infty$, and do not exceed values of order one. This means that in the specific basis we have chosen, $\left\{\omega_{\alpha}\right\}=\left\{J_{0}, C_{i}\right\}, B^{0}$ is the only 2 -form gauge field that becomes weakly coupled as $t \rightarrow \infty$, while all other linear combinations of fields $B^{i}$ become strongly coupled.

To make contact with (2.18) and (2.19), we fist recall that the split of 2-form potentials into self-dual and anti-self-dual tensors can be found by diagonalizing the duality matrix, ${ }^{6}$

$$
D_{J}^{I}:=\left(g^{-1}\right)^{I K} \Omega_{K J}=\frac{1}{\mathcal{V}}\left(J \cdot C^{I}\right)\left(J \cdot C_{J}\right)-\delta^{I}{ }_{J},
$$

which obeys

$$
* H^{I}=D_{J}^{I} H^{J}
$$

Then, in an appropriate basis of curves, $\left\{C^{\prime I}\right\}$, the duality matrix can be taken in a diagonal form,

$$
D^{\prime I}=\operatorname{diag}(+1,-1,-1, \cdots,-1) .
$$

Upon a further change of basis one can find another basis of curves, $\left\{C^{\prime \prime I}\right\}$, with respect to which the duality matrix takes the form

$$
D_{J}^{\prime I I}=\left(\begin{array}{cc|ccc}
0 & S^{-2} & 0 & \cdots & 0 \\
S^{2} & 0 & 0 & \cdots & 0 \\
\hline 0 & 0 & -1 & \cdots & 0 \\
\vdots & \vdots & \vdots & \ddots & \vdots \\
0 & 0 & 0 & \cdots & -1
\end{array}\right) .
$$

Correspondingly, the set of 2-forms, $\left\{B^{\prime \prime I}\right\}$, comprises $\mathrm{T}, \tilde{\mathrm{T}}$, plus the remaining anti-selfdual 2-forms, $B_{i=2, \cdots, n_{T}}^{(-)}$. The form of (2.30) guarantees that in this latter basis,

$$
C^{\prime \prime 0}=C^{0}+\mathcal{O}(1 / t):=J_{0}+\mathcal{O}(1 / t) .
$$

We can hence identify the weakly coupled tensor and its associated coupling parameter as

$$
\mathrm{T}=B^{0}+\mathcal{O}(1 / t), \quad S=\frac{t}{\sqrt{\mathcal{V}}} .
$$

\footnotetext{
${ }^{6} \mathrm{~A}$ detailed discussion of the split of 2 -form potentials into self-dual and anti-self-dual tensors can be found in section 2 of [57].
} 
Most importantly, the string from the D3-brane wrapping $C^{0}$ is identified with a weakly coupled string, $\mathcal{S}$, whose tension satisfies the relation

$$
\frac{T_{\mathcal{S}}}{M_{\mathrm{Pl}}^{2}}=\sqrt{\pi} S^{-1}
$$

The existence of this tensionless string in the weak coupling limit is in agreement with expectation from the Swampland Distance Conjecture. Even though we have not explicitly constructed it in full generality, it is clear that the D3-brane on the curve $C^{\prime \prime 1}$ gives rise to a dual string, $\tilde{S}$, with tension

$$
\frac{T_{\tilde{\mathcal{S}}}}{M_{\mathrm{Pl}}^{2}}=\sqrt{\pi} S
$$

This can be confirmed by investigating explicit examples.

\subsection{Emergent heterotic string at weak coupling}

As discussed in our previous work [11], the correct interpretation of the tensionless string is that it takes the role of the weakly coupled heterotic string in a new duality frame, that is:

$$
\text { F-theory on } B_{2} \quad \text { limit } \stackrel{(2.21)}{\Longrightarrow},(2.22) \quad \text { Heterotic on K3 with } S_{\text {het }}^{2}:=\mathcal{V}_{\text {het }} e^{-\Phi_{\text {het }}}=\frac{t^{2}}{\mathcal{V}}
$$

Indeed, an analysis of the $N=(0,4)$ supersymmetric worldsheet theory of the solitonic string from the D3-brane wrapped on $C^{0}$ identifies it with the critical heterotic string compactified to six dimensions [11] on a certain K3 surface. This realizes the well-known phenomenon that the critical heterotic string can be viewed as a soliton [58] and serves as the microscopic rationale behind standard F-theory-heterotic duality [49-51]. The novel insight of our analysis is that a weak coupling limit for any 2-form in F-theory inevitably realises a version of this duality: in the limit (2.21) the theory switches duality frame from the original Type IIB/F-theory frame to a dual heterotic frame, where the role of the fundamental string is played by the solitonic string associated with $C^{0}$.

This implies that in the duality frame defined by the weakly coupled string, the specific linear combination $\mathrm{T}=B^{0}$ of 2 -forms to which this string couples plays the role of the "universal" 2-form Kalb-Ramond field B that couples to the fundamental string. To set the notation, recall that dimensional reduction to six dimensions on $\mathrm{K} 3$ of the heterotic 10d Einstein frame action (with $\ell_{\text {het }} \equiv 1$ ) yields

$$
S_{\text {het }, 6 \mathrm{~d}}^{(E)}=\int_{\mathbb{R}^{1,5}}\left(\frac{M_{\mathrm{Pl}}^{4}}{2} \sqrt{-g} R-\frac{2 \pi}{4}\left(S_{\text {het }}^{2} d \mathrm{~B} \wedge * d \mathrm{~B}+S_{\text {het }}^{-2} d \tilde{\mathrm{B}} \wedge * d \tilde{\mathrm{B}}\right)\right)
$$

where

$$
M_{\mathrm{Pl}}^{4}=4 \pi \mathcal{V}_{\text {het }}, \quad S_{\text {het }}^{2}=\mathcal{V}_{\text {het }} e^{-\Phi_{\text {het }}} .
$$

We have written the kinetic terms in a democratic fashion by introducing the "magnetically" dual field, $* d \tilde{\mathrm{B}}=S_{\text {het }}^{2} d \mathrm{~B}$. It couples to a dual string which arises as an NS5-brane wrapping the K3 on the heterotic side. Since we have kept the physical Planck mass fixed in taking the weak coupling limit on the F-theory side, the volume of the dual heterotic 
K3 surface emerging as the weakly coupled description must equal the volume of $B_{2}$ on the F-theory side,

$$
\mathcal{V}_{\text {het }}=\mathcal{V}
$$

The identification (2.40) then follows by identifying the tension of the fundamental heterotic string in the above frame,

$$
\frac{T_{F_{\text {het }}}}{M_{\mathrm{Pl}}^{2}}=\sqrt{\pi} \frac{e^{\Phi_{\text {het }} / 2}}{\sqrt{\mathcal{V}}}=\sqrt{\pi} S_{\text {het }}^{-1},
$$

with the tension of the asymptotically weakly coupled string, $\mathcal{S}$, obtained on the F-theory side:

$$
S=S_{\text {het }} \quad \rightarrow \quad e^{\Phi_{\text {het }}}=\frac{\mathcal{V}^{2}}{t^{2}} .
$$

To summarize, whenever we take a weak coupling limit for some (linear combination of) 2-form potentials in F-theory, which we denote by $B^{0} \equiv \mathrm{T}$, while keeping gravity dynamical, a tensionless, weakly coupled string $\mathcal{S}$ arises, which is dual to a heterotic string propagating in six dimensions. The 2 -form potential under which this string is charged is precisely the 2 -form $B^{0}$ which becomes weakly coupled. The theory is then best described by switching the duality frame to the frame where the asymptotically weakly coupled string acts as the fundamental string, and where $B^{0}$ takes the role of the heterotic Kalb-Ramond 2 -form potential.

Since in the weak coupling limit the heterotic string becomes tensionless, all its excitation modes become massless. Integrating out these modes reproduces the perturbative supergravity effective action. In this sense, the running of the coupling constants in the effective action is emergent: it can either be understood from the perspective of the original supergravity theory, or by integrating out the full tower of asymptotically light modes arising from the emergent fundamental (here, heterotic) string.

Note that in addition to the modes of the nearly tensionless string, the theory potentially contains an extra tower of Kaluza-Klein modes, which are simply the Kalzua-Klein modes of the nearly tensionless string along any submanifold that simultanously happens to become large in the limit (2.21). We will elaborate further on this point at the end of section 3.4, where we will encounter a similar emergence of a fundamental string, together with a Kalzua-Klein tower, in the weak coupling limit of a different theory.

\section{Emergent fundamental strings from Type IIB strings on K3}

In this section we analyze the weak coupling limit for 2 -form potentials of Type IIB string theory compactified on an "attractive" K3 surface. ${ }^{7}$ Our main result is summarised in

Claim 2 Consider a certain limit where a 2-form gauge potential in Type IIB string theory compactified on an attractive K3 becomes asymptotically weakly coupled, while gravity is kept dynamical. In this limit, there emerges an asymptotically tensionless solitonic string which is charged under the weakly coupled 2-form potential. Via duality it corresponds to a critical Type IIB string propagating on an elliptic K3 surface.

\footnotetext{
${ }^{7}$ We will explain this notion further below.
} 
Thus, in a sense the theory reproduces itself in the duality frame relevant at infinite distance in moduli space, which is of considerable significance for the proposal of emergence $[2,10,25-27]$.

\subsection{Large distance limits in the moduli space $\mathcal{M}_{(5,21)}$}

Type IIB string theory compactified on a K3 surface is approximated, at low energies, by an effective 6 d supergravity theory with $N=(2,0)$ supersymmetry. Many important properties of K3 surfaces as probed by Type IIB string theory can be found in [59] and references therein. The bosonic part of the gravity multiplet of $6 \mathrm{~d} N=(2,0)$ supergravity contains the metric degrees of freedom as well as five self-dual tensors $B_{i}^{(+)}, i=1, \ldots 5$, which transform as $\mathbf{5}$ of the R-symmetry group $\operatorname{Sp}(4)$. The remaining degrees of freedom organize into tensor multiplets, each of which containing, at the bosonic level, one anti-self dual tensor along with 5 real scalars. For Type IIB string theory on K3, there are altogether 21 such tensor multiplets, into which the 105 moduli fields organize. This 105 dimensional, nonperturbative moduli space of Type IIB string theory on K3 can be written as the coset space

$$
\mathcal{M}_{(5,21)}=O\left(\Gamma_{5,21}\right) \backslash O(5,21) / O(5) \times O(21),
$$

where $O\left(\Gamma_{5,21}\right)$ represents the discrete $U$-duality group of the theory.

The total of 26 self-dual or anti-self-dual tensor fields of the theory arise as follows: the 10d Kalb-Ramond field $B_{2}$ and the Ramond-Ramond cousin $C_{2}$ give rise to a $6 \mathrm{~d}$ tensor field each, which is neither self-dual nor anti-self-dual and is accompanied by its respective dual counterpart, $\tilde{B}_{2}$ and $\tilde{C}_{2}$. In addition, the theory contains $h^{2}(K 3)=22$ massless self-dual or anti-self-dual tensor fields, $B^{A}$, which arise from dimensional reduction of the Ramond-Ramond 4-form $C_{4}$ field via

$$
C_{4}=B^{A} \wedge \omega_{A}, \quad \omega_{A} \in H^{2}(K 3, \mathbb{R}), \quad A=1, \ldots, 22 .
$$

Our interest is in the general structure of the possible weak coupling limits for the tensor fields at infinite distance in moduli space. The moduli space (3.1) contains five noncompact directions, which we parametrize by moduli $S_{i}, i=1, \ldots, 5$. Moving to infinite distance along any of these five directions gives rise to a generally different weak coupling limit. ${ }^{8}$ Each of the five non-compact scalars $S_{i}$ sits in a separate tensor multiplet. The associated anti-self-dual 2-forms, $B_{i}^{(-)}, i=1, \ldots, 5$, pair up with the 5 self-dual tensors in the gravity multiplet, $B_{i}^{(+)}$, to produce a six-dimensional 2 -form tensor field $\mathrm{T}_{i}$ (which is neither self-dual nor anti-self-dual), plus its dual, $\tilde{\mathrm{T}}_{i}$. In a given weak coupling limit parametrized by $S_{i}$, the tensor field $\mathrm{T}_{i}$ becomes weakly coupled (resp. its dual, $\tilde{\mathrm{T}}_{i}$, in the opposite limit).

More specifically, let us fix our notation by taking the kinetic terms for the given weakly coupled system of tensors to be

$$
S_{6 \mathrm{~d}}=\int_{\mathbb{R}^{1,5}}\left(\frac{1}{2} M_{\mathrm{Pl}}^{4} \sqrt{-|g|} R-\frac{2 \pi}{4}\left(S_{i}^{2} d \mathrm{~T}_{i} \wedge * d \mathrm{~T}_{i}+S_{i}^{-2} d \tilde{\mathrm{T}}_{i} \wedge * d \tilde{\mathrm{T}}_{i}\right)+\ldots\right)
$$

\footnotetext{
${ }^{8}$ Note that for taking such limits, one must specify how precisely the boundary of $\mathcal{M}_{(5,21)}$ is approached. We will come back to this point below.
} 


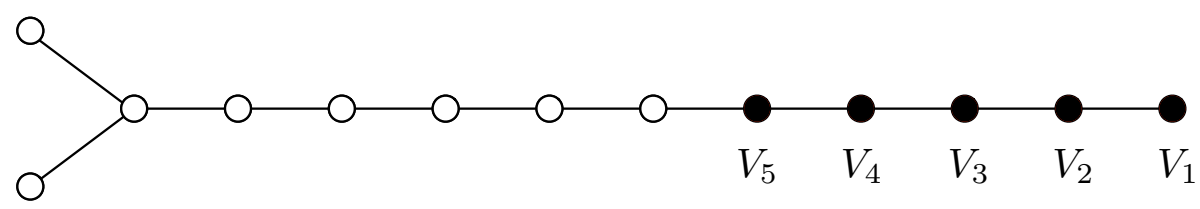

Figure 1. Dynkin Diagram of the $U$-duality group $\operatorname{SO}(5,21)$, which encodes the non-perturbative moduli space $\mathcal{M}_{(5,21)}$, as per ref. [60]. The black dots denote five non-compact directions in which one can take large distance limits. The directions $V_{1}$ and $V_{2}$ correspond to the standard limits of weak $10 \mathrm{~d}$ dilaton and large volume, respectively. Note that the basis of the $V_{i}$ is not the same as the basis of $S_{i}$ which correspond to the weak coupling limits of 2 -forms that we consider.

with

$$
* d \mathrm{~T}_{i}=S_{i}^{-2} d \tilde{\mathrm{T}}_{i}
$$

and no significant kinetic mixing of the weakly coupled tensor $T_{i}$ with the other tensor fields in the limit $S_{i} \rightarrow \infty$. This corresponds to the conventions

$$
\begin{array}{rll}
S_{i} \rightarrow \infty: & \mathrm{T}_{i} \text { becomes weakly coupled }, & i=1, \ldots, 5, \\
S_{i}^{-1} \rightarrow \infty: & \tilde{\mathrm{T}}_{i} \text { becomes weakly coupled }, & i=1, \ldots, 5 .
\end{array}
$$

As $S_{i} \rightarrow \infty$, the string $\mathcal{S}_{i}$ to which $\mathrm{T}_{i}$ couples "electrically" becomes tensionless, while the dual string $\tilde{\mathcal{S}}_{i}$, to which $\tilde{\mathrm{T}}_{i}$ couples, becomes heavy. That is, the scalar $S_{i}$ is related to the tension of the string $\mathcal{S}_{i}$ that couples electrically to $\mathrm{T}_{i}$ in the following way:

$$
\begin{aligned}
& T_{\mathcal{S}_{i}} / M_{\mathrm{Pl}}^{2}=\sqrt{\pi} S_{i}^{-1} \\
& T_{\tilde{\mathcal{S}}_{i}} / M_{\mathrm{Pl}}^{2}=\sqrt{\pi} S_{i} .
\end{aligned}
$$

The division by the squared Planck mass normalizes the tension of the strings with respect to the six-dimensional Planck scale, and the numerical factors are chosen such that the tension of the string and its dual satisfy the canonical relation

$$
T_{\mathcal{S}_{i}} T_{\tilde{\mathcal{S}}_{i}}=\pi M_{\mathrm{Pl}}^{4} .
$$

The possible non-compact limits of the moduli space $\mathcal{M}_{(5,21)}$ in (3.1) have been analyzed in [60] in terms of splitting the Dynkin diagram of $\mathrm{SO}(5,21)$ into pieces by removing the respective relevant black dots [61] (see figure 1). This analysis mostly focused on the resulting moduli spaces but not on tensionless strings that may arise in the various limits. The point of our work is to analyze the appearance of tensionless strings from the viewpoint of dually weakly coupled tensionless Type IIB strings, in agreement with the idea of emergence.

For two out of these five possible weak coupling limits, the appearance of tensionless strings is evident, because these limits are nothing but the standard limits for the sixdimensional 2-form tensor fields that descend from the various ten-dimensional tensor fields. More specifically, let us fix our conventions by starting from the ten-dimensional action in the Einstein frame, with $\ell_{s} \equiv 1$,

$$
S_{10 \mathrm{~d}}^{\mathrm{E}}=2 \pi \int_{\mathbb{R}^{1,9}}\left(\sqrt{-|g|} R-\frac{1}{2} e^{-\Phi} d B_{2} \wedge * d B_{2}-\frac{1}{2} e^{\Phi} d C_{2} \wedge * d C_{2}-\frac{1}{4} d C_{4} \wedge * d C_{4}+\ldots\right),
$$


in terms of which the tensions of the various branes read

$$
\begin{aligned}
& T_{D p}=2 \pi e^{\Phi\left(\frac{p+1}{4}-1\right)}, \quad \Phi: 10 \mathrm{~d} \text { Einstein frame dilaton } \\
& T_{F 1}=2 \pi e^{\Phi / 2}, \quad T_{N S 5}=2 \pi e^{-\Phi / 2} .
\end{aligned}
$$

Then, after compactifying on a K3 surface with volume $\mathcal{V}$, the relevant part of the action is

$$
\begin{gathered}
S_{6 \mathrm{~d}}^{\mathrm{E}}=\int_{\mathbb{R}^{1,5}}\left(\frac{1}{2} M_{\mathrm{Pl}}^{4} \sqrt{-|g|} R-\frac{2 \pi}{4}\left(S_{2}^{2} d B_{2} \wedge * d B_{2}+S_{2}^{-2} d \tilde{B}_{2} \wedge * d \tilde{B}_{2}\right)\right. \\
\left.-\frac{2 \pi}{4}\left(S_{1}^{2} d C_{2} \wedge * d C_{2}+S_{1}^{-2} d \tilde{C}_{2} \wedge * d \tilde{C}_{2}\right)+\ldots\right)
\end{gathered}
$$

with

$$
M_{\mathrm{Pl}}^{4}=4 \pi \mathcal{V}, \quad S_{2}=e^{-\Phi / 2} \sqrt{\mathcal{V}}, \quad S_{1}=e^{\Phi / 2} \sqrt{\mathcal{V}}
$$

We have expressed the kinetic terms in a democratic fashion by employing the dual fields $\tilde{B}_{2}$ and $\tilde{C}_{2}$, which arise from the ten-dimensional six-form fields, $B_{6}$ and $C_{6}$, and which satisfy:

$$
* d B_{2}=S_{2}^{-2} d \tilde{B}_{2}, \quad * d C_{2}=S_{1}^{-2} d \tilde{C}_{2} .
$$

The properly normalised tensions ${ }^{9}$ of the $F 1$ and $D 1$ strings and of their magnetic duals, $\tilde{F} 1$ and $\tilde{D} 1$, are then

$$
\begin{array}{ll}
\frac{T_{F 1}}{M_{\mathrm{Pl}}^{2}}=\sqrt{\pi} \frac{e^{\Phi / 2}}{\sqrt{\mathcal{V}}} \equiv \sqrt{\pi} S_{2}^{-1} & \frac{T_{\tilde{F} 1}}{M_{\mathrm{Pl}}^{2}}=\sqrt{\pi} \frac{\sqrt{\mathcal{V}}}{e^{\Phi / 2}} \equiv \sqrt{\pi} S_{2}, \\
\frac{T_{D 1}}{M_{\mathrm{Pl}}^{2}}=\sqrt{\pi} \frac{1}{e^{\Phi / 2} \sqrt{\mathcal{V}}} \equiv \sqrt{\pi} S_{1}^{-1} & \frac{T_{\tilde{D} 1}}{M_{\mathrm{Pl}}^{2}}=\sqrt{\pi} e^{\Phi / 2} \sqrt{\mathcal{V}} \equiv \sqrt{\pi} S_{1} .
\end{array}
$$

Thus $S_{2} \rightarrow \infty$ is the weak coupling limit for the six-dimensional field $B_{2}$ (or strong coupling limit for its dual $\tilde{B}_{2}$ ), as follows from the kinetic terms in (3.13). Consistently, the fundamental string $\mathcal{S}_{2} \equiv F 1$ becomes asymptotically tensionless, while its "magnetically" dual string, $\tilde{F} 1$, becomes heavy and decouples. On the other hand, the tension of the $D 1 / \tilde{D} 1$ string system, compared to the $F 1 / \tilde{F} 1$ string system, depends on the priori independent value of

$$
e^{\Phi} \equiv \frac{S_{1}}{S_{2}}=: V_{1}
$$

If we keep $S_{1}$ fixed and finite, we have that $e^{\Phi} \equiv \frac{S_{1}}{S_{2}} \ll 1$ as $S_{2} \rightarrow \infty$, and so the F1 string is indeed the lightest string and can be properly viewed as the fundamental Type IIB string.

Conversely, in the weak coupling limit of the field $C_{2}$, where $S_{1} \rightarrow \infty$, the string $\mathcal{S}_{1} \equiv D 1$ becomes light and eventually tensionless, while its dual $\tilde{D} 1$ decouples. As long as we are in the geometric regime, where the volume

$$
\mathcal{V} \equiv S_{1} S_{2}=: V_{2}
$$

\footnotetext{
${ }^{9}$ Alternatively we can preform a $6 \mathrm{~d}$ Weyl rescaling to bring the Einstein-Hilbert term into the form $\frac{1}{2} \int_{\mathbb{R}^{1,5}} \sqrt{-g} R$. The resulting rescaling of the string tensions corresponds to division by $M_{\mathrm{P} 1}^{2}$ in (3.17). Note furthermore that $\Phi$ refers to the dilaton in the 10d Einstein frame.
} 
is fixed and large, the D1-string is furthermore parametrically lighter than the string $\tilde{F} 1$, while its dual, $F 1$, decouples.

The two limits, $S_{1} \rightarrow \infty$ or $S_{2} \rightarrow \infty$, are of course S-dual to each other, in the sense of ten-dimensional SL $(2, \mathbb{Z})$ symmetry: the ten-dimensional S-duality transformation maps $\Phi \rightarrow-\Phi$ in the 10d Einstein frame dilaton, and hence acts as interchange

$$
\text { 10d S-duality: } \quad S_{1} \leftrightarrow S_{2}
$$

It is important to note that in order to properly define infinite distance limits, one must precisely specify in which way variables become large, ie., how their ratios behave. Mathematically this is tied to the notion of a proper compactification of the moduli space, $\overline{\mathcal{M}}_{(5,21)}$. In the present work, we consider limits for which the volume of K3 stays fixed in order to keep gravity dynamical, which is of importance for our investigation of the Swampland Distance Conjectures for 2-form fields. This amounts to considering the limits $S_{i} \rightarrow \infty$, as opposed to products thereof such as (3.20), as it is the $S_{i}$ which canonically couple to the 2 -form fields.

As is evident from (3.19) and (3.20), this basis of non-compact moduli is different from the basis of the $V_{i}$ which are tied to the eponymous nodes in the Dynkin diagram in figure 1. The $V_{i}$ figure in the discussion [60] of how the moduli space $\mathcal{M}_{(5,21)}$ splits up upon taking non-compact limits by removing dots in the Dynkin diagram. These limits are different to the less singular limits in the $S_{i}$ that we take, and correspond to a different compactification of the moduli space.

More precisely, in the infinite distance limit where $V_{1}=S_{1} / S_{2} \rightarrow 0$, we recover the weakly coupled perturbative string on $\mathrm{K} 3$, whose moduli space is the moduli space of $N=(4,4)$ supersymmetric sigma-models on K3. The latter is given by $\mathcal{M}_{(4,20)}=$ $O(4,20) / O(4) \times O(20)$ (modulo discrete identifications), which is obtained from the full moduli space (3.1) by removing the node $V_{1}$ in the Dynkin diagram of figure 1 [60]. Similarly, for $V_{2}=S_{1} S_{2} \rightarrow \infty$, one obtains the 10d Type IIB string compactified on a large volume K3, whose moduli space contains $\mathcal{M}_{(3,19) \times(1,1)}=O(3,19) /(O(3) \times O(19)) \times$ $\mathrm{SU}(1,1) / \mathrm{U}(1)$. The first factor is the geometric moduli space of a K3 surface of fixed volume and the second is the non-perturbative moduli space of the eight-dimensional Type IIB string. Note that this large volume limit is compatible with the $\operatorname{SL}(2, \mathbb{Z})$ action (3.21).

\subsection{Geometric weak coupling limits in Kähler moduli space of K3}

The upshot of the previous section is that two of the five possible weak coupling limits of Type IIB string theory on K3 have a straightforward explanation that involves only combinations of the $10 \mathrm{~d}$ dilaton $e^{\Phi}$ and the overall volume $\mathcal{V}$ of K3. The remaining three weak coupling limits must correspond to non-trivial degeneration limits of the K3 surface as such. We will analyze one of these in detail now, and make the relation between the weak coupling regime and the appearance of a solitonic tensionless string, which will turn out to be a Type IIB string again. 
The remaining weak coupling limits must involve three combinations of the 22 tensor fields $B^{A}$ from decomposing $C_{4}$ as in (3.2), with kinetic terms

$$
S_{\mathrm{kin}}=-\frac{2 \pi}{4} \int_{\mathbb{R}^{1,5}} g_{A B} d B^{A} \wedge * d B^{B}
$$

governed by

$$
g_{A B}=\int_{K 3} \omega_{A} \wedge * \omega_{B} .
$$

It is convenient to pick an arbitrary complex structure on $\mathrm{K} 3$ and consider the resulting Hodge decomposition of $H^{2}(K 3, \mathbb{R})$. For any such chosen complex structure, the Hodge numbers of $\mathrm{K} 3$ are

$$
h^{(2,0)}(K 3)=1, \quad h^{(1,1)}(K 3)=20, \quad h^{(0,2)}(K 3)=1 .
$$

Let us specify a basis of 2-forms by

$$
\left\{\omega_{A}\right\}=\left\{\Omega, \bar{\Omega}, \omega_{\alpha}\right\}, \quad \omega_{\alpha} \in H^{(1,1)}(K 3), \quad \Omega \in H^{(2,0)}(K 3) .
$$

Here $\Omega$ and $\bar{\Omega}$ denote the unique $(2,0)$ form and its complex conjugate.

The space $H^{2}(K 3, \mathbb{R})$ can also be decomposed into spaces of self-dual and anti-self-dual 2 -forms with respect to the Hodge star operator on K3,

$$
\begin{aligned}
H^{2}(K 3, \mathbb{R}) & =H_{+}^{2}(K 3, \mathbb{R}) \oplus H_{-}^{2}(K 3, \mathbb{R}), \\
\operatorname{dim} H_{+}^{2}(K 3, \mathbb{R}) & =3, \quad \operatorname{dim} H_{-}^{2}(K 3, \mathbb{R})=19 .
\end{aligned}
$$

The space of self-dual 2-forms is spanned by the real and imaginary parts of $\Omega$ and the Kähler form $J$. According to the discussion of the previous section, the tensors which become weakly coupled in the three remaining weak coupling limits must involve linear combinations of the self-dual tensors associated with reduction of $C_{4}$ along these three directions in $H_{+}^{2}(K 3, \mathbb{R})$, together with three anti-self-dual tensors form expansion of $C_{4}$ along three directions in $H_{-}^{2}(K 3, \mathbb{R})$.

In the given complex structure, one of the weak coupling limits therefore involves only a tensor field and its dual associated with the $(1,1)$ forms on K3. It is this weak coupling limit on which we focus in the sequel. ${ }^{10}$ On the space $H^{1,1}(K 3)$, the matrix of kinetic terms of the 2-forms $B^{\alpha}$ takes the form

$$
g_{\alpha \beta}=\frac{1}{\mathcal{V}} j_{\alpha} j_{\beta}-\Omega_{\alpha \beta},
$$

where $J=j^{\alpha} \omega_{\alpha}$ and $\Omega_{\alpha \beta}=\omega_{\alpha} \cdot \omega_{\beta}$. The weak coupling limit for the system of tensormultiplet 2 -forms is therefore characterized by demanding that the kinetic matrix $g_{\alpha \beta}$ has at least one entry which tends to infinity.

Note that the Kähler form implicitly depends on the choice of complex structure with respect to which the Hodge type of the 2 -forms is defined. In this sense, the matrix $g_{\alpha \beta}$

\footnotetext{
${ }^{10}$ The remaining two infinite distance limits necessarily involve complex structure deformations, which are beyond the scope of our analysis.
} 
depends on all geometric moduli of the $\mathrm{K} 3$ and not only on the moduli entering $J$. A related peculiarity of K3 surfaces is that the rank of the Picard group

$$
\operatorname{Pic}\left(K_{3}\right)=H^{1,1}(K 3) \cap H_{2}(K 3, \mathbb{Z})
$$

depends on the choice of complex structure. The elements of the Picard group describe the curve classes $H_{2}(K 3, \mathbb{Z})$ which have a holomorphic representative.

A simplification occurs when the rank of $\operatorname{Pic}\left(K_{3}\right)$ takes its maximal value: $\operatorname{rkPic}\left(K_{3}\right)=$ 20. This means that all curve classes have holomorphic representatives, and their volume reduces to the Kähler volume with respect to the Kähler form $J$ of the K3 surface, viewed as a Kähler manifold with vanishing anti-canonical bundle $\bar{K}=0$. Such K3 surfaces are referred to as singular K3s in mathematics, and as attractive K3s in the physics literature [62]. Attractive K3 surfaces lie dense in the period domain of K3 surfaces, in a similar way as $\mathbb{Q}^{n}$ lies dense in $\mathbb{R}^{n}$. The complex structure of an attractive K3 is completely fixed. In particular, the kinetic matrix (3.28) depends solely on the Kähler moduli of the attractive K3. We will for now focus on such K3 surfaces.

On an attractive K3, the weak coupling limit for (3.28) takes a completely analogous form as for the F-theory two-fold bases, which were discussed in section 2. Indeed in order for at least one eigenvalue of (3.28) to become large, the Kähler form $J$ of the compact K3 must asymptote to

$$
J=t J_{0}+\sum_{i} \frac{a_{i}}{2 t} J_{i} \quad t \rightarrow \infty, \quad \text { subject to }(2.22)
$$

In the basis

$$
\left\{C^{I}\right\}=\left\{C^{0}:=J_{0}, \quad C^{i}:=J_{i}\right\}
$$

the matrix of kinetic terms takes the asymptotic form (2.30) and (2.31), and the unique 2 -form potential which becomes weakly coupled is precisely $B^{0}$. It couples to the solitonic string that arises from wrapping a D3-brane on the holomorphic curve $C^{0}$. The latter has the property

$$
C^{0} \cdot C^{0}=0
$$

and moreover its volume vanishes in the weak coupling limit,

$$
\operatorname{vol}\left(C^{0}\right)=\int_{C^{0}} J=\frac{\mathcal{V}}{t} \quad \text { as } \quad t \rightarrow \infty
$$

Indeed, since the Kähler cone generators are dual to the Mori cone of effective curves, they are by themselves integral $(1,1)$ classes, and on an attractive K3 every such class has a representative as a holomorphic curve. The novelty for this limit for K3, as compared to a Kähler base $B_{2}$ with non-trivial anti-canonical bundle, is that the genus of $C^{0}$ equals

$$
g\left(C^{0}\right)=1+\frac{1}{2}\left(C^{0} \cdot C^{0}-C^{0} \cdot \bar{K}\right)=1 .
$$


The existence of a class $C^{0} \in H^{1,1}(K 3) \cap H_{2}(K 3, \mathbb{Z})$ with vanishing self-intersection implies that the $\mathrm{K} 3$ surface is a genus-one fibration, with fiber $C^{0}$, i.e. there exists a projection

$$
\begin{array}{rr}
\pi: \quad C^{0} \rightarrow & K 3 \\
& \downarrow \\
& C_{b} .
\end{array}
$$

See e.g. [63] for a proof of this theorem, which is the analogue of Kollar's conjecture for K3 surfaces. ${ }^{11}$

We can summarize these findings by stating that, in the notation of section 3.1 , the tensor field

$$
\mathrm{T}_{3}:=B^{0}
$$

is singled out as the tensor which is weakly coupled in the geometric limit $t \rightarrow \infty$. Its kinetic terms can asymptotically be written as

$$
S_{\text {kin }}=-\frac{2 \pi}{4} \int_{\mathbb{R}^{1,5}}\left(S_{3}^{2} d B^{0} \wedge * d B^{0}+S_{3}^{-2} d \tilde{B}^{0} \wedge * d \tilde{B}^{0}\right)
$$

with

$$
S_{3}=\frac{t}{\sqrt{\mathcal{V}}}
$$

If we also introduce the notation

$$
\mathcal{S}_{3}:=\text { String from a D3 - brane on } C^{0},
$$

then the normalised tension of $\mathcal{S}_{3}$ scales as

$$
T_{\mathcal{S}_{3}} / M_{\mathrm{Pl}}^{2}=\sqrt{\pi} \frac{\sqrt{\mathcal{V}}}{t}=: \sqrt{\pi} S_{3}^{-1} .
$$

In (3.37) we have exhibited also the magnetically dual field $\tilde{B}^{0} \equiv \tilde{\mathrm{T}}_{3}$. Correspondingly the solitonic string $\mathcal{S}_{3}$ has a magnetic dual $\tilde{\mathcal{S}}_{3}$ arising from a D3-brane wrapping a dual cycle on K3. This cycle can be obtained in principle by carefully going through the procedure outlined at the end of section 2.2: we need to bring the duality matrix associated with the kinetic matrix (2.30) obtained in the limit into the form (2.35), which conforms with (3.37). Let us denote the cycle associated with $\tilde{B}^{0}$ as $C^{\prime \prime 1}$, as in section 2.2. Even without determining its cycle class explicitly we anticipate from the expected tension of the dual string that its volume must scale as

$$
\operatorname{vol}_{\tilde{C}^{\prime \prime 1}} \sim t .
$$

Clearly such cycles are present in the Picard lattice: in the limit (3.30) this is the behaviour of a cycle with

$$
J_{0} \cdot C^{\prime \prime 1} \neq 0 .
$$

This string becomes heavy and strongly coupled in the limit $t \rightarrow \infty$ for fixed $\mathcal{V}$, and effectively decouples.

\footnotetext{
${ }^{11}$ This can be seen as a physical proof for the geometrical statement that attractive K3 surfaces are genusone fibered, under the assumption that weak coupling limits exist in the Kähler moduli space. Indeed, it is an established fact in mathematics that a K3 surface with Picard number $\rho$ is genus-one fibered if $\rho \geq 5$ and is elliptic with a section if $\rho \geq 13$; see e.g. [64].
} 


\begin{tabular}{|c|c|c|c|c|c|}
\hline \multicolumn{2}{|c|}{ Fermions } & \multicolumn{2}{|c|}{ Bosons } & $N=(4,4)$ & Multiplicity \\
\hline$(\mathbf{2}, \mathbf{1})_{1}+$ c.c. & $\psi_{+}+$c.c. & $(\mathbf{1}, \mathbf{1})_{0},(\mathbf{1}, \mathbf{1})_{0}$ & $\bar{a}, \bar{\sigma}$ & Hyper & $h^{0,1}\left(C^{0}\right)=g=1$ \\
$(\mathbf{1}, \mathbf{2})_{-1}+$ c.c. & $\tilde{\rho}_{-}+$c.c. & $(\mathbf{1}, \mathbf{1})_{0},(\mathbf{1}, \mathbf{1})_{0}$ & $a, \sigma$ & & \\
\hline$(\mathbf{1}, \mathbf{2})_{1}+$ c.c. & $\mu_{+}+$c.c. & $(\mathbf{2}, \mathbf{2})_{0}$ & $\varphi$ & Vector & $h^{0,0}\left(C^{0}\right)=1$ \\
$(\mathbf{2}, \mathbf{1})_{-1}+$ c.c. & $\lambda_{-}+$c.c. & $(\mathbf{1}, \mathbf{1})_{ \pm 2}$ & $v_{ \pm}$ & & \\
\hline
\end{tabular}

Table 1. Spectrum of massless $2 \mathrm{~d} N=(4,4)$ multiplets on the worldsheet of a string obtained by wrapping a D3-brane on the genus-one curve $C^{0} \subset K 3$ with $C^{0} \cdot C^{0}=0$. The first column indicates the representations with respect to $\mathrm{SU}(2)_{L} \times \mathrm{SU}(2)_{R} \times \mathrm{SO}(1,1)$. We denote by $+/-$ the $2 \mathrm{~d}$ left/right-moving chiralities.

\subsection{The solitonic string as a Type IIB fundamental string}

The tensionless string $\mathcal{S}_{3}$, in fact, describes a critical, fundamental Type IIB string propagating on $\mathbb{R}^{1,5} \times K 3$. This follows from (3.34) in view of the findings of [65], which we now briefly review from the perspective of our purposes.

The worldsheet theory of $\mathcal{S}_{3}$ is derived via dimensional reduction of the worldvolume theory on a single D3-brane wrapped on the genus-one curve $C^{0}$. The latter is, of course, given by $4 \mathrm{~d} N=4$ Super-Yang-Mills theory with gauge group $\mathrm{U}(1)$. Compactification on $C^{0} \subset K 3$ breaks one half of the 16 supercharges on the D3-brane and results in a string worldsheet theory with $N=(4,4)$ supersymmetry.

This can be made manifest by performing a standard topological twist along $C^{0}$ : the $\mathrm{SU}(4)_{R}$ symmetry of the D3-brane theory, which is identified with the $\mathrm{SO}(6)_{T}$ rotation group acting on the dimensions transverse to the D3-brane, decomposes as

$$
\mathrm{SO}(6)_{T} \rightarrow \mathrm{SO}(4)_{T} \times \mathrm{U}(1)_{R}, \quad \mathrm{SO}(4)_{T}=\mathrm{SU}(2)_{L} \times \mathrm{SU}(2)_{R}
$$

where $\mathrm{SO}(4)_{T}$ acts on the four extended directions $\mathbb{R}_{T}^{4}$ normal to the string. The Lorentz group parallel to the D3-brane decomposes into

$$
\mathrm{SO}(1,3) \rightarrow \mathrm{SO}(1,1) \times \mathrm{U}(1)_{C}
$$

where the two factors act on the extended and internal dimensions of the string, respectively. The topological twist is defined with respect to the combination

$$
\mathrm{U}(1)_{\mathrm{tw}}=\frac{1}{2}\left(\mathrm{U}(1)_{C}+\mathrm{U}(1)_{R}\right)
$$

Only the supercharges which are singlets under this $\mathrm{U}(1)_{\mathrm{tw}}$ are preserved. Twisted dimensional reduction of the $N=4$ vector multiplet leads to one $N=(4,4)$ hypermultiplet along with one $N=(4,4)$ vector multiplet, as displayed in table $1 .{ }^{12}$

Following the arguments of [67], in the limit of vanishing curve volume for $C^{0}$ the gauge kinetic term for the $\mathrm{U}(1)$ potential $v_{ \pm}$along the string decouples and the worldsheet theory

\footnotetext{
${ }^{12}$ More details can be found e.g. in section 3.1.1 of [66]. This reference performs, in addition to the standard topological twist, a duality twist, which must be omitted for K3 with trivial canonical bundle.
} 
reduces to a non-linear sigma model. If we ignore the non-dynamical vector field $v_{ \pm}$, the field content agrees precisely with that of a critical, fundamental Type II string propagating on $\mathbb{R}^{1,5} \times K 3$ : the four real scalars $\varphi$ transform as a vector under $\mathrm{SO}(4)_{T}$ and describe the fluctuations in the transverse directions $\mathbb{R}_{T}^{4} \subset \mathbb{R}^{1,5}$. Together with their superpartners they are associated with a free sector of the worldsheet theory. The remaining four real scalars $a, \bar{a}, \sigma, \bar{\sigma}$ and their superpartners are the fundamental fields of an interacting non-linear sigma model. Two of these, $a$ and $\bar{a}$, are associated with the string motion along $C^{0}$, while $\sigma$ and $\bar{\sigma}$ describe the fluctuations in the internal directions normal to $C^{0}$.

The target space of the of non-linear sigma model is identified with the moduli space of the string [67]. Apart from the external directions transverse to the string, this moduli space coincides precisely the original manifold K3 [65]. To see this, one makes use of the fact that the wrapped curve $C^{0}$ is the elliptic fiber of K3, according to (3.35). The moduli space of $C^{0}$ as a holomorphic curve is identical to the base $C_{b}$ over which it is fibered. In addition, the sigma-model moduli space includes the moduli space of flat gauge backgrounds on a D3-brane on $C^{0}$. The latter are described by the Jacobian of the curve, which gives back the curve itself (or rather its dual). As a result, one recovers the full fibration of $C^{0}$ over $C_{b}$ as the internal part of the moduli space of the string on $C^{0}$ [65]. Together with the external part this yields a non-linear sigma-model on $\mathbb{R}_{T}^{4} \times K 3$.

\subsection{Emergent strings and duality in the geometric weak coupling limit}

The previous considerations make it evident that, as we take the large distance, weak coupling limit (3.30), we can switch to a new duality frame, denoted by hatted quantities in the sequel. The solitonic string $\mathcal{S}_{3}$ (D3 brane wrapped on $C_{0}$ ) in the original frame turns into the weakly coupled, fundamental Type IIB string $\widehat{F} 1 \equiv \widehat{\mathcal{S}}_{2}$ propagating on $K 3$. The weakly coupled 2-form field $\mathrm{T}_{3}:=B^{0}$ with coupling $S_{3}$ given in (3.40) in the old frame takes the role of the fundamental field $\widehat{T}_{2} \equiv \hat{B}_{2}$ in the new frame, with corresponding 'dilaton' $\widehat{S}_{2}=S_{3}$ :

$$
\text { Type IIB on K3 } \stackrel{\operatorname{limit}(3.30)}{\Longrightarrow} \text { Type IIB on K3 with } \widehat{S}_{2}^{2}:=\mathcal{V} e^{-\widehat{\Phi}}=\frac{t^{2}}{\mathcal{V}}
$$

As in the analogous F-theory/heterotic correspondence we are identifying the volume of the original theory with the volume of the new theory to the extent that we insist on keeping the Planck scale fixed.

In the duality frame defined by the string $\mathcal{S}_{3}=\widehat{\mathcal{S}}_{2}$, there must also arise a heavy S-dual string $\widehat{D} 1 \equiv \widehat{\mathcal{S}}_{1}$. Its associated coupling $\widehat{S}_{1}$ follows from the coupling $\widehat{S}_{2}=S_{3}$ by demanding that

$$
\widehat{S}_{1} \widehat{S}_{2}=\widehat{\mathcal{V}} \equiv \mathcal{V}
$$

in analogy to the standard relation (3.20) for the S-dual tensions in the weakly coupled fundamental frame. Together with (3.46) this gives

$$
\widehat{S}_{1}^{-1}=\frac{t}{\mathcal{V}^{3 / 2}} .
$$

Such a string can arise from a D3-brane wrapping a curve $\Sigma$ on K3 with

$$
\operatorname{vol}(\Sigma)=\frac{t}{\mathcal{V}}
$$


The magnetic dual of this string, $\widehat{\tilde{D}}_{1} \equiv \widehat{\tilde{\mathcal{S}}}_{1}$ has normalised tension

$$
\frac{T_{\widehat{\mathcal{S}}_{1}}}{M_{\mathrm{Pl}}^{2}}=\sqrt{\pi} \widehat{S}_{1}=\sqrt{\pi} \frac{\mathcal{V}^{3 / 2}}{t}=\mathcal{V} \frac{T_{\widehat{\mathcal{S}}_{2}}}{M_{\mathrm{Pl}}^{2}},
$$

which vanishes in the limit $t \rightarrow \infty$, even though it is enhanced compared to the tension of the new fundamental string $\mathcal{S}_{3} \equiv \widehat{\mathcal{S}}_{2}$ by a factor of $\mathcal{V}$.

It is interesting to wonder which curve $\Sigma$ can give rise to the behaviour (3.49). All curves in the Picard lattice obtain their volume exclusively from the intersection with the Kähler form $J$. As pointed out already, the volumes of such curves which become large in the limit $t \rightarrow \infty$ are the ones with non-zero overlap with the Kähler cone generator $J_{0}$, but their volumes scale as $t$ as opposed to $t / \mathcal{V}$. This argument seems to suggest that the curve $\Sigma$ obtains its volume rather from the overlap with the $(2,0)$ form $\Omega$, in the sense of a symplectic integral. A natural conjecture is therefore that the S-dual curve lies in the overlap of $\left(H^{2,0}(K 3) \oplus H^{0,2}(K 3)\right) \cap H_{2}(K 3, \mathbb{Z})$, or at least receives contributions from this space. Note that for attractive K3s, this space is 2-dimensional [68].

An important point to keep in mind is that the moduli space of six-dimensional theories with $N=(2,0)$ supersymmetry is not corrected by worldsheet and D1-instanton effects. Hence even though Euclidean F1 and D1 strings (of the original duality frame) wrapped on the vanishing cycle(s) on K3 might look like giving rise to a non-suppressed instanton effect, they will not affect the classical couplings in the limit we are considering. The magnetic duals of such instantons in six dimensions are $(3+1)$-dimensional objects in $\mathbb{R}^{1,5}$ and correspond to the original D5 or NS5-brane wrapping curves on K3. For the vanishing curve $C^{0}$, the tension of these objects scales as $T_{3+1} \sim \operatorname{vol}\left(C^{0}\right)=\mathcal{V} / t$ and hence vanishes at the same time as the tension of the new fundamental string $\widehat{\mathcal{S}}_{2}$ goes to zero. However, the associated mass scales compare as

$$
M_{3+1}=T_{3+1}^{1 / 4} \sim\left(\frac{\mathcal{V}}{t}\right)^{1 / 4} \gg\left(\frac{\mathcal{V}}{t}\right)^{1 / 2} \sim T_{\widehat{\mathcal{S}}_{2}}^{1 / 2}=M_{\widehat{\mathcal{S}}_{2}} \Longrightarrow \frac{M_{3+1}}{M_{\widehat{\mathcal{S}}_{2}}} \sim\left(\frac{\mathcal{V}}{t}\right)^{-1 / 4} \rightarrow \infty
$$

Hence these objects decouple in the duality frame defined by the new fundamental string $\widehat{\mathcal{S}}_{2}$.

Even though the string $\mathcal{S}_{3}$ takes the role of the new fundamental string $\widehat{S}_{2}$ on K3, the limit $t \rightarrow \infty$ at fixed $\mathcal{V}$, i.e. $S_{3} \rightarrow \infty$ at fixed $\mathcal{V}$, is different from the weak coupling limit $S_{2} \rightarrow \infty$ at fixed $\mathcal{V}$ studied in section 3.1. The reason is that in addition to the tower of excitations from $\mathcal{S}_{3}$ which becomes massless, there is a tower of asymptotically light KaluzaKlein (KK) states which arises because we are considering Type IIB on a K3 in a very special deformation limit. The origin of the KK tower are the cycles which become large in the limit (3.30), i.e. all curves with $C_{\text {large }} \cdot J_{0} \neq 0$. Since their volume scales as $\operatorname{vol}\left(C_{\text {large }}\right) \sim t$, the mass scale of the Kaluza-Klein excitations associated with these curves is

$$
M_{\mathrm{KK}}^{2} \sim \operatorname{vol}\left(C_{\text {large }}\right)^{-1}=\frac{1}{t} \sim \frac{T_{\mathcal{S}_{3}}}{\mathcal{V}} \equiv \frac{T_{\widehat{\mathcal{S}}_{2}}}{\mathcal{V}}
$$

The states with mass scale $M_{\mathrm{KK}}$ are the Kaluza-Klein excitations of the new fundamental string $\widehat{\mathcal{S}}_{2}$ in the new Type IIB frame. This is consistent in that $\widehat{\mathcal{S}}_{2}$ arises from a D3-brane 
wrapped on the elliptic fiber $C^{0}$ of K3, and it therefore makes sense to consider the KalzuaKlein spectrum of its excitations, at each excitation level, along the remaining directions on K3.

Let us contrast the limits $S_{3} \rightarrow \infty$ versus $S_{2} \rightarrow \infty$ as follows:

$$
\begin{aligned}
& \text { Limit } S_{2} \rightarrow \infty, \mathcal{V} \gg 1 \text { fixed : } \quad e^{\Phi} \rightarrow 0 \\
& \qquad\left(M_{\mathcal{S}_{2}}^{2}=e^{\Phi / 2}\right)<\left(M_{\tilde{\mathcal{S}}_{1}}^{2}=e^{\Phi / 2} \mathcal{V}\right)<\left(M_{K K}^{2}=\frac{1}{\sqrt{\mathcal{V}}}\right)<\left(M_{\mathrm{Pl}}^{2}=\sqrt{\mathcal{V}}\right) \\
& \text { Limit } S_{3} \rightarrow \infty, \mathcal{V} \gg 1 \text { fixed }: \quad \frac{1}{t} \rightarrow 0 \\
& \qquad\left(M_{\mathrm{KK}}^{2}=\frac{1}{t}\right)<\left(M_{\mathcal{S}_{2}}^{2}=\frac{\mathcal{V}}{t}\right)<\left(M_{\tilde{S}_{1}}^{2}=\frac{\mathcal{V}^{2}}{t}\right)<\left(M_{\mathrm{Pl}}^{2}=\sqrt{\mathcal{V}}\right)
\end{aligned}
$$

The interesting feature of the geometric weak coupling limit (3.54) is that for fixed $\mathcal{V}$, the KK excitations sit at the same parametric scale as the excitations of $\widehat{\mathcal{S}}_{2}$ itself, as far as the scaling with the weak coupling parameter $t$ is concerned. In particular, as the new string tension $T_{\widehat{\mathcal{S}}_{2}}=\frac{2 \pi \mathcal{V}}{t}$ asymptotes to zero, so does the KK scale, albeit relatively suppressed by a factor of $\mathcal{V}$. Hence the limit $t \rightarrow \infty$ governs both the asymptotically massless tower of oscillator excitations of the new fundamental weakly coupled string, $\widehat{\mathcal{S}}_{2}$, as well as simultaneously its Kaluza-Klein tower of states. In both situations (3.53) and (3.54) there arises in addition the tower of the magnetic version of the S-dual string, which is enhanced by another factor of $\mathcal{V}$. Despite the appearance of the KK tower in the limit (3.54) we would not call this a decompactification limit in the usual sense: such a limit is detected by a encountering only a tower of light KK states, without an accompanying tower of extra string modes.

The observation that the tower of massless states here includes the tower of a fundamental string sheds some light on the proposal of emergence put forward in [2, 10, 25-27]: according to the general lore, in the weak coupling limit at infinite field distance, a tower of states becomes massless exponentially fast [3]; integrating out this tower of states leads to the running of the coupling constants of the theory and reproduces the polynomial singularity at infinite distance in moduli space, as encountered in the effective supergravity description. Evidence for this proposal has been provided in [10, 11, 14, 25-27] by estimating, at a qualitative level, the contribution of the integrated tower of particle states to the renormalization of the couplings. On the other hand, an exact computation reproducing the observed supergravity coupling constants near infinite distance has not been obtained in the literature, as it is a priori much more difficult to quantify the spectrum of the asymptotically massless states.

What we are observing here is that the massless tower of states has a very clear interpretation as exactly the excitations of the critical fundamental string $\widehat{\mathcal{S}}_{2}$ in the new duality frame plus its Kaluza-Klein excitations. Importantly, the new fundamental string is again the Type IIB string probing the same K3 (in the specific geometric limit). Integrating out the entire tower of string excitations together with the KK modes reproduces, by construction, the coupling dependence of the original supergravity theory in the weak coupling limit. This is true not only parametrically, but at an exact level, by the very 
definition of the low-energy effective supergravity as the consequence of integrating out the heavy string states and the Kaluza-Klein/winding modes on the internal space. In this sense, even without explicitly evaluating this integration, it is manifest that the polynomial divergence in the coupling constants at infinite distance is exactly reproduced and, in fact, caused by the tower of states which become asymptotically massless.

\section{Emergent F-theory from M-theory on K3 in weak coupling limit}

As we have shown in the previous sections, following refs. [11, 12], weak coupling limits in the Kähler moduli space of F-theory and Type IIB compactifications lead to emergent critical, nearly tensionless strings along with their towers of oscillator and Kaluza-Klein excitations. As we will show in this section, the same geometrical limits in Kähler moduli that we have analyzed above have a strikingly different effect as probed by M-theory or Type IIA string theory. A detailed analysis of infinite distance limits in Kähler moduli space from the perspective of Type IIA/M-theory has been provided before in [14], and we will comment on the relation of this work to our findings below.

Consider thus M-theory compactified on a K3 surface to $\mathrm{d}=7$ dimensions, which leaves 16 supercharges unbroken. The crucial difference to the situation in F-theory or Type IIB theory is that this theory contains $h^{2}(K 3)=221$-form gauge fields, as opposed to 2-form fields, 3 of which are part of the gravity multiplet. These 1-form fields are obtained by the reduction of the M-theory 3 -form $C_{3}$ :

$$
C_{3}=A^{\alpha} \wedge \omega_{\alpha} \quad \omega_{\alpha} \in H^{1,1}(K 3) .
$$

The same coupling matrix of kinetic terms as in (3.28) now applies to the 1-form gauge fields $A^{\alpha}$, as opposed to the 2 -forms $B^{\alpha}$ in Type IIB/F-theory.

For simplicity, let us restrict ourselves to attractive K3 surfaces with a Picard group of maximal rank, as in the previous section. Then, despite the different interpretation, the analysis of the weak coupling limit at fixed $7 \mathrm{~d}$ Planck scale proceeds in an entirely identical fashion: it corresponds to taking the limit

$$
J=t J_{0}+\sum_{i} \frac{a_{i}}{2 t} J_{i}, \quad t \rightarrow \infty, \quad \text { subject to }(2.22) .
$$

In the basis of $(1,1)$-forms $\left\{\omega_{\alpha}\right\}=\left\{J_{0}, J_{i}\right\}$, the gauge field $A^{0}$ is the only linear combination of 1 -form potentials which becomes asymptotically weakly coupled as $t \rightarrow \infty$. The pertinent gauge kinetic matrix can be found in (2.30).

Thus, a unique genus one curve $C^{0}:=J_{0}$ shrinks at the rate $\operatorname{vol}\left(C^{0}\right)=\frac{\mathcal{V}}{t}$. What becomes massless in the weak coupling limit is now a tower of particles (as opposed to a string and its excitations), which arise from M2-branes wrapping the shrinking curve $C^{0}$. Importantly, the Gopakumar-Vafa invariants for an $n$-fold wrapped genus-one curve on K3 are [65]

$$
N_{n \cdot C^{0}}^{\mathrm{GV}}=24 \quad \forall n \geq 1 .
$$

This fact guarantees the existence of a tower of asymptotically massless states in the effective theory. 
As we have discussed around eq. (3.35), in order for the K3 to admit the weak coupling limit (4.2), it must be fibered with a genus one curve $C^{0}$,

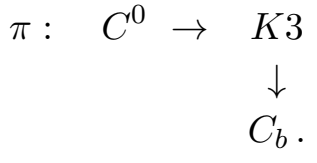

The weak coupling limit hence amounts to shrinking the genus one fiber of the K3 while keeping its total volume fixed. This limit coincides exactly with the F-theory limit associated with M-theory on K3:

$$
\begin{array}{lll}
\text { F-theory on } C_{b} \times S^{1} & \Longleftrightarrow & \begin{array}{l}
\text { M-theory on K3 as in (4.4) } \\
S^{1} \text {-radius } R
\end{array}
\end{array}
$$

Recall that F-theory on a genus-one fibered K3-surface over the base $C_{b}$ gives rise to an eight-dimensional theory with 16 supercharges. Upon compactification on a circle $S^{1}$ with radius $R$, we obtain a $7 \mathrm{~d}$ theory which is identified with M-theory on the same K3, in the limit where the fiber volume scales as $\frac{1}{R}$. Each $8 \mathrm{~d}$ field maps to a full Kaluza-Klein tower of excitations in the $7 \mathrm{~d}$ M-theory, which are in turn interpreted as M2-branes wrapping the fiber $C^{0} n$-times. These M2-branes are charged under the Kaluza-Klein gauge potential $A_{\mathrm{KK}}$.

We therefore see that the weak coupling limit for an - a priori arbitrary - 1-form gauge field in M-theory on K3, inevitably enforces the F-theory limit: the asymptotically weakly coupled gauge field, $A^{0}$ is identified with the Kaluza-Klein U(1) KK gauge group in the F-theory/M-theory correspondence, and the tower of asymptotically massless particles from M2-branes along the curve $n \cdot C^{0}$ represent the Kaluza-Klein tower associated with the $8 \mathrm{~d}$ supergravity modes of F-theory.

Hence, we encounter an emergent extra dimension in the weak coupling limit, in which the duality frame of the theory switches from that of $7 \mathrm{~d}$ M-theory to the duality frame of 8d F-theory:

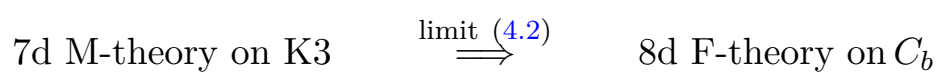

Importantly, the gauge fields in the F-theory are not in a weak coupling regime. Rather the weakly coupled M-theory 1-form has become part of the $8 \mathrm{~d}$ metric, since it refers to the U(1) gauge field associated with the Kaluza-Klein reduction.

Note that infinite distance limits in Kähler moduli space and their interpretation in Type IIA and M-theory have been analyzed in detail in [14]. This reference includes a study of infinite distance limits on elliptically fibered Calabi-Yau 3-folds, and, as a special case of such limits, the F-theory limit of vanishing fiber volume. The key point of our discussion is that whenever one considers a weak coupling limit in the Kähler moduli space, on an attractive K3, the shrinking curve is automatically a genus one fiber of the K3, and the weak coupling limit reduces to the F-theory limit, even without making any assumption about the fibration structure of the K3 surface. In this sense the emergence of the extra dimension in F-theory is a consequence of the weak coupling limit. 


\section{Conclusions, and prospects for four-dimensional strings}

In this work we have explored the behavior of effective theories near infinite distance points in moduli space where 2-form gauge fields become weakly coupled, in the presence of gravity. Specifically we have systematically analyzed such weak coupling limits for sixdimensional compactifications of F-theory and Type IIB theory, with $N=(1,0)$ and $N=$ $(2,0)$ supersymmetry, respectively. Two universal phenomena have emerged in the limit:

1) A critical string becomes tensionless, be it the fundamental string in the original duality frame or, generically, a solitonic string which takes the role of the fundamental string in a dual frame.

2) An infinite tower of asymptotically massless particles arise as the excitations of the emergent critical string, in agreement with the Swampland Distance Conjecture.

For F-theory compactifications, we have obtained the general form of the weak coupling limits of 2 -forms. The limit turns out to be identical to the weak coupling limits of 1 -form gauge fields studied in [11], in the Kähler moduli space of the internal complex surface. By the same arguments as in [11] a solitonic string emerges as a critical, weakly coupled heterotic string.

On the other hand, for Type IIB compactifications, the moduli space has five different types of non-compact directions. Two obvious directions correspond to the limits where the ten-dimensional Kalb-Ramond and Ramond-Ramond 2-forms are weakly coupled, and hence where the fundamental string or the D1 string becomes tensionless, respectively. Amongst the remaining three 'geometric' weak coupling limits, we have systematically analyzed the one reachable within the Kähler moduli space of the internal K3 surfaces, upon restricting to geometries with maximal Picard number $\rho=20$. We have singled out a unique elliptic curve class with non-negative normal bundle whose volume vanishes in the weak coupling limit. A D3 brane wrapping this curve leads to an emergent tensionless string, which we have identified as a weakly coupled Type IIB string in yet another Type IIB duality frame. Its quantum excitations give rise to an infinite tower of massless particles in the limit. The remaining two non-compact weak coupling limits lie in the complex structure moduli space and we leave their further investigation to future work.

An interesting aspect of weak coupling limits in the Kähler moduli space, for both F-theory and Type IIB compactifications, is that another set of particles become asymptotically massless, in addition to the tower arising from the quantization of the relevant, nearly tensionless string. These are the Kaluza-Klein modes that become light in the geometric limits we consider. The masses of these KK modes exhibit the same suppression factor by the large Kähler parameter as the string modes, although an additional suppression by the volume, $\mathcal{V}$, of the K3 is inevitable. Since the KK tower becomes massless at the same rate as the string tower, the limit should not be interpreted as the unfolding of a new dimension. We have contrasted this situation with the weak coupling limits of 1-form potentials in M-theory compactifications on K3 surfaces, once again restricting the discussion to geometries with maximal Picard number. In such limits, the fiber class of 
an elliptic fibration shrinks, uplifting the seven-dimensional M-theory compactification to F-theory in eight dimensions.

While this work has focused on effective theories in $d=6$ dimensions, many of our conclusions pertaining the nature of tensionless strings carry over to four dimensions, as we now briefly discuss: for the four-dimensional compactifications of both F-theory and Type IIB theory, the 2 -form fields $B^{I}$ arise from expanding $C_{4}$ over a basis $C_{I}$ of $H^{1,1}\left(B_{3}\right)$ and have the kinetic coupling matrix

$$
g_{I J}=-\frac{1}{4 \mathcal{V}} C_{I} \cdot C_{J} \cdot J+\left(\frac{1}{4 \mathcal{V}}\right)^{2}\left(C_{I} \cdot J \cdot J\right)\left(C_{J} \cdot J \cdot J\right)
$$

where $\mathcal{V}=\frac{1}{6} \int_{B_{3}} J^{3}$ is the volume of the internal three-fold $B_{3}$. This $B_{3}$ is the base of an elliptic four-fold in F-theory, while on the other hand it coincides with the internal Calabi-Yau three-fold when we talk about a Type IIB string compactification. In the weak coupling limit, at least one entry of $g_{I J}$ diverges (and hence at least one of the Kähler parameters goes to infinity) while $\mathcal{V}$ is kept fixed. Geometric limits of this kind have been considered in section 6.1 of [15] and classified as limits of Class A and Class B, respectively.

In the weak coupling limits of Class A, the Kähler form takes the following non-negative expansion in terms of the Kähler cone generators,

$$
J=t J_{0}+\sum_{\nu} \frac{a_{\nu}}{t^{2}} J_{\nu}+\sum_{r} c_{r} J_{r}, \quad \text { with } J_{0} \cdot J_{0} \neq 0, \quad t \rightarrow \infty
$$

where $J_{0} \cdot J_{0} \cdot J_{0}=0$ and $J_{0} \cdot J_{0} \cdot J_{\nu}>0$ for $\nu$ in a certain index set, while $J_{0} \cdot J_{0} \cdot J_{r}=0$ and $J_{0} \cdot J_{r} \cdot J_{s}=0$ for $r$ and $s$ in another index set. It was then proven that there exists a curve $C^{0}:=J_{0} \cdot J_{0}$ with a trivial normal bundle which shrinks in such a geometric limit. For F-theory compactifications, as argued in [15], such a curve has to be a rational curve and hence a D3 brane wrapping $C^{0}$ plays the role of the tensionless critical heterotic string in the heterotic duality frame.

If $B_{3}$ is itself a Calabi-Yau three-fold, on the other hand, the existence of the Kähler cone generator $J_{0}$ implies [69-71] that $B_{3}$ is necessarily genus-one fibered; the shrinking curve $C^{0}$ is the fiber of this fibration. The world-sheet theory associated with a D3-brane wrapped on $C^{0}$ can be determined by methods similar to those spelled out in [66]: the result is a world-sheet theory with $N=(2,2)$ supersymmetry. Its bosonic excitations include two real scalars parametrizing the motion of the string in the two extended directions transverse to the string, two complex scalars transforming as sections of the normal bundle $N_{C^{0} / B_{3}}=\mathcal{O} \oplus \mathcal{O}$ to the wrapped curve and one complex scalar transforming as a section of $\mathcal{O}_{C^{0}}$, corresponding to the Wilson line degrees of the freedom. The moduli space of the D3-brane along $C^{0}$ is the fibration of $C^{0}$ over the base of the fibration, and hence coincides with $B_{3}$. In the limit of shrinking curve volume we therefore manifestly arrive at a sigma-model with target space $B_{3}$, which we interpret as the critical Type IIB string propagating on $B_{3}$. By the same logic as in section 3 of the present paper, we conclude that the emerging tensionless string is the Type IIB string in another Type IIB duality frame. The theory, again, reproduces itself in the dual weak coupling limit at infinite distance. 
It is intriguing to see that the Calabi-Yau three-fold is necessarily elliptic in order to admit a weak coupling limit of the form (5.2). For five-dimensional M-theory compactifications, the existence of such a geometric limit corresponds to a weak coupling limit for 1-form potentials. As for M-theory on K3 analysed in this paper, we can immediately conclude that a weak coupling limit of Class A for 5-dimensional M-theory is a decompactification limit which coincides with the F-theory uplift to six dimensions. This is a consequence of the properties of the limit (5.2) and holds without making any a priori assumptions about the existence of an elliptic fibration.

The situation is more complicated for the weak coupling limits of Class B [15], however, which we leave to future work.

In view of these results in the context of F-theory and Type IIB theory, it is natural to wonder about the nature of the tensionless strings that emerge in weak coupling limits for 2-forms in Type IIA compactifications. Six-dimensional Type IIA compactifications on K3 surfaces do not have 2-forms which can become weakly coupled in the geometric moduli space. On the other hand, in four-dimensional Type IIA compactifications on Calabi-Yau three-folds 2-forms arise from expanding either the Ramond-Ramond 5-forms over harmonic 3-forms or the dual 6-form of the Kalb-Ramond 2-form over harmonic 4forms. These respectively admit weak coupling limits in the complex structure and Kähler moduli space $[10,13,14,18]$ of the Calabi-Yau three-folds. Mirror symmetry suggests that analogous phenomena as in the Type IIB case should occur. The pressing question in this context is whether the resulting nearly tensionless strings are dual to some weakly coupled strings, similarly to what we have found here for six-dimensional compactifications of F-theory and Type IIB theory.

\section{Acknowledgments}

We thank Yang-Hui He, Fernando Marchesano, Eran Palti, Christian Reichelt, Fabian Rühle, Max Wiesner and Fengjun Xu for helpful discussions. The work of SJL is supported by the Korean Research Foundation (KRF) through the CERN-Korea Fellowship program.

Open Access. This article is distributed under the terms of the Creative Commons Attribution License (CC-BY 4.0), which permits any use, distribution and reproduction in any medium, provided the original author(s) and source are credited.

\section{References}

[1] T.D. Brennan, F. Carta and C. Vafa, The string landscape, the swampland, and the missing corner, PoS TASI2017 (2017) 015 [arXiv:1711.00864] [INSPIRE].

[2] E. Palti, The swampland: introduction and review, Fortsch. Phys. 67 (2019) 1900037 [arXiv: 1903. 06239] [INSPIRE].

[3] H. Ooguri and C. Vafa, On the geometry of the string landscape and the swampland, Nucl. Phys. B 766 (2007) 21 [hep-th/0605264] [INSPIRE].

[4] N. Arkani-Hamed, L. Motl, A. Nicolis and C. Vafa, The string landscape, black holes and gravity as the weakest force, JHEP 06 (2007) 060 [hep-th/0601001] [INSPIRE]. 
[5] B. Heidenreich, M. Reece and T. Rudelius, Evidence for a sublattice weak gravity conjecture, JHEP 08 (2017) 025 [arXiv:1606.08437] [INSPIRE].

[6] M. Montero, G. Shiu and P. Soler, The weak gravity conjecture in three dimensions, JHEP 10 (2016) 159 [arXiv: 1606.08438] [InSPIRE].

[7] D. Klaewer and E. Palti, Super-Planckian spatial field variations and quantum gravity, JHEP 01 (2017) 088 [arXiv:1610.00010] [INSPIRE].

[8] B. Heidenreich, M. Reece and T. Rudelius, Sharpening the weak gravity conjecture with dimensional reduction, JHEP 02 (2016) 140 [arXiv:1509.06374] [INSPIRE].

[9] S. Andriolo, D. Junghans, T. Noumi and G. Shiu, A tower weak gravity conjecture from infrared consistency, Fortsch. Phys. 66 (2018) 1800020 [arXiv: 1802.04287] [INSPIRE].

[10] T.W. Grimm, E. Palti and I. Valenzuela, Infinite distances in field space and massless towers of states, JHEP 08 (2018) 143 [arXiv: 1802.08264] [INSPIRE].

[11] S.-J. Lee, W. Lerche and T. Weigand, Tensionless strings and the weak gravity conjecture, JHEP 10 (2018) 164 [arXiv:1808.05958] [INSPIRE].

[12] S.-J. Lee, W. Lerche and T. Weigand, A stringy test of the scalar weak gravity conjecture, Nucl. Phys. B 938 (2019) 321 [arXiv: 1810.05169] [INSPIRE].

[13] T.W. Grimm, C. Li and E. Palti, Infinite distance networks in field space and charge orbits, JHEP 03 (2019) 016 [arXiv:1811.02571] [INSPIRE].

[14] P. Corvilain, T.W. Grimm and I. Valenzuela, The swampland distance conjecture for Kähler moduli, JHEP 08 (2019) 075 [arXiv: 1812.07548] [INSPIRE].

[15] S.-J. Lee, W. Lerche and T. Weigand, Modular fluxes, elliptic genera, and weak gravity conjectures in four dimensions, JHEP 08 (2019) 104 [arXiv:1901.08065] [INSPIRE].

[16] A. Joshi and A. Klemm, Swampland distance conjecture for one-parameter Calabi-Yau threefolds, JHEP 08 (2019) 086 [arXiv:1903.00596] [INSPIRE].

[17] F. Marchesano and M. Wiesner, Instantons and infinite distances, JHEP 08 (2019) 088 [arXiv: 1904.04848] [INSPIRE].

[18] A. Font, A. Herráez and L.E. Ibáñez, The swampland distance conjecture and towers of tensionless branes, JHEP 08 (2019) 044 [arXiv: 1904.05379] [INSPIRE].

[19] E. Gonzalo, L.E. Ibáñez and A.M. Uranga, Modular symmetries and the swampland conjectures, JHEP 05 (2019) 105 [arXiv:1812.06520] [INSPIRE].

[20] F. Baume and E. Palti, Backreacted axion field ranges in string theory, JHEP 08 (2016) 043 [arXiv: 1602.06517] [INSPIRE].

[21] R. Blumenhagen, D. Kläwer, L. Schlechter and F. Wolf, The refined swampland distance conjecture in Calabi-Yau moduli spaces, JHEP 06 (2018) 052 [arXiv: 1803.04989] [INSPIRE].

[22] H. Ooguri, E. Palti, G. Shiu and C. Vafa, Distance and de Sitter conjectures on the swampland, Phys. Lett. B $\mathbf{7 8 8}$ (2019) 180 [arXiv:1810.05506] [INSPIRE].

[23] G. Obied, H. Ooguri, L. Spodyneiko and C. Vafa, De Sitter space and the swampland, arXiv: 1806.08362 [INSPIRE].

[24] A. Strominger, Massless black holes and conifolds in string theory, Nucl. Phys. B 451 (1995) 96 [hep-th/9504090] [INSPIRE]. 
[25] D. Harlow, Wormholes, emergent gauge fields, and the weak gravity conjecture, JHEP 01 (2016) 122 [arXiv:1510.07911] [INSPIRE].

[26] B. Heidenreich, M. Reece and T. Rudelius, The weak gravity conjecture and emergence from an ultraviolet cutoff, Eur. Phys. J. C 78 (2018) 337 [arXiv:1712.01868] [InSPIRE].

[27] B. Heidenreich, M. Reece and T. Rudelius, Emergence of weak coupling at large distance in quantum gravity, Phys. Rev. Lett. 121 (2018) 051601 [arXiv:1802.08698] [INSPIRE].

[28] T. Rudelius, On the possibility of large axion moduli spaces, JCAP 04 (2015) 049 [arXiv:1409.5793] [INSPIRE].

[29] A. de la Fuente, P. Saraswat and R. Sundrum, Natural inflation and quantum gravity, Phys. Rev. Lett. 114 (2015) 151303 [arXiv: 1412.3457] [INSPIRE].

[30] T. Rudelius, Constraints on axion inflation from the weak gravity conjecture, JCAP 09 (2015) 020 [arXiv:1503.00795] [INSPIRE].

[31] M. Montero, A.M. Uranga and I. Valenzuela, Transplanckian axions!?, JHEP 08 (2015) 032 [arXiv: 1503.03886] [INSPIRE].

[32] J. Brown, W. Cottrell, G. Shiu and P. Soler, Fencing in the swampland: quantum gravity constraints on large field inflation, JHEP 10 (2015) 023 [arXiv:1503.04783] [INSPIRE].

[33] T.C. Bachlechner, C. Long and L. McAllister, Planckian axions and the weak gravity conjecture, JHEP 01 (2016) 091 [arXiv: 1503.07853] [INSPIRE].

[34] A. Hebecker, P. Mangat, F. Rompineve and L.T. Witkowski, Winding out of the swamp: evading the weak gravity conjecture with F-term winding inflation?, Phys. Lett. B 748 (2015) 455 [arXiv: 1503.07912] [INSPIRE].

[35] J. Brown, W. Cottrell, G. Shiu and P. Soler, On axionic field ranges, loopholes and the weak gravity conjecture, JHEP 04 (2016) 017 [arXiv: 1504.00659] [INSPIRE].

[36] B. Heidenreich, M. Reece and T. Rudelius, Weak gravity strongly constrains large-field axion inflation, JHEP 12 (2015) 108 [arXiv:1506.03447] [INSPIRE].

[37] K. Kooner, S. Parameswaran and I. Zavala, Warping the weak gravity conjecture, Phys. Lett. B 759 (2016) 402 [arXiv:1509.07049] [INSPIRE].

[38] L.E. Ibáñez, M. Montero, A. Uranga and I. Valenzuela, Relaxion monodromy and the weak gravity conjecture, JHEP 04 (2016) 020 [arXiv:1512.00025] [INSPIRE].

[39] A. Hebecker, F. Rompineve and A. Westphal, Axion monodromy and the weak gravity conjecture, JHEP 04 (2016) 157 [arXiv: 1512.03768] [INSPIRE].

[40] B. Heidenreich, M. Reece and T. Rudelius, Axion experiments to algebraic geometry: testing quantum gravity via the weak gravity conjecture, Int. J. Mod. Phys. D 25 (2016) 1643005 [arXiv: 1605.05311] [INSPIRE].

[41] R. Blumenhagen, I. Valenzuela and F. Wolf, The swampland conjecture and F-term axion monodromy inflation, JHEP 07 (2017) 145 [arXiv: 1703.05776] [INSPIRE].

[42] I. Valenzuela, Backreaction in axion monodromy, 4-forms and the swampland, PoS CORFU2016 (2017) 112 [arXiv: 1708.07456] [INSPIRE].

[43] L.E. Ibáñez and M. Montero, A note on the WGC, effective field theory and clockwork within string theory, JHEP 02 (2018) 057 [arXiv: 1709.02392] [INSPIRE]. 
[44] G. Aldazabal and L.E. Ibáñez, A note on $4 D$ heterotic string vacua, FI-terms and the swampland, Phys. Lett. B $\mathbf{7 8 2}$ (2018) 375 [arXiv:1804.07322] [INSPIRE].

[45] R. Blumenhagen, Large field inflation/quintessence and the refined swampland distance conjecture, PoS CORFU2017 (2018) 175 [arXiv:1804.10504] [INSPIRE].

[46] M. Reece, Photon masses in the landscape and the swampland, JHEP 07 (2019) 181 [arXiv: 1808.09966] [INSPIRE].

[47] A. Hebecker and P. Soler, The weak gravity conjecture and the axionic black hole paradox, JHEP 09 (2017) 036 [arXiv: 1702.06130] [INSPIRE].

[48] M. Montero, A.M. Uranga and I. Valenzuela, A Chern-Simons pandemic, JHEP 07 (2017) 123 [arXiv: 1702.06147] [INSPIRE].

[49] C. Vafa, Evidence for F-theory, Nucl. Phys. B 469 (1996) 403 [hep-th/9602022] [InSPIRE].

[50] D.R. Morrison and C. Vafa, Compactifications of F-theory on Calabi-Yau threefolds. 1, Nucl. Phys. B 473 (1996) 74 [hep-th/9602114] [INSPIRE].

[51] D.R. Morrison and C. Vafa, Compactifications of F-theory on Calabi-Yau threefolds. 2, Nucl. Phys. B 476 (1996) 437 [hep-th/9603161] [InSPIRE].

[52] E. Gonzalo and L.E. Ibáñez, A strong scalar weak gravity conjecture and some implications, JHEP 08 (2019) 118 [arXiv: 1903.08878] [INSPIRE].

[53] E. Palti, The weak gravity conjecture and scalar fields, JHEP 08 (2017) 034 [arXiv: 1705.04328] [INSPIRE].

[54] W. Taylor, TASI lectures on supergravity and string vacua in various dimensions, arXiv:1104.2051 [INSPIRE].

[55] T. Weigand, F-theory, PoS TASI2017 (2018) 016 [arXiv:1806. 01854] [INSPIRE].

[56] F. Bonetti and T.W. Grimm, Six-dimensional $(1,0)$ effective action of F-theory via M-theory on Calabi-Yau threefolds, JHEP 05 (2012) 019 [arXiv:1112.1082] [INSPIRE].

[57] S.-J. Lee, D. Regalado and T. Weigand, 6d SCFTs and U(1) flavour symmetries, JHEP 11 (2018) 147 [arXiv: 1803.07998] [INSPIRE].

[58] J.A. Harvey and A. Strominger, The heterotic string is a soliton, Nucl. Phys. B 449 (1995) 535 [Erratum ibid. 458 (1996) 456] [hep-th/9504047] [INSPIRE].

[59] P.S. Aspinwall, K3 surfaces and string duality, in Theoretical Advanced Study Institute in Elementary Particle Physics (TASI 96): fields, strings, and duality, (1996), pg. 421 [hep-th/9611137] [INSPIRE].

[60] P.S. Aspinwall and D.R. Morrison, U duality and integral structures, Phys. Lett. B 355 (1995) 141 [hep-th/9505025] [INSPIRE].

[61] E. Witten, String theory dynamics in various dimensions, Nucl. Phys. B 443 (1995) 85 [hep-th/9503124] [INSPIRE].

[62] G.W. Moore, Arithmetic and attractors, hep-th/9807087 [INSPIRE].

[63] D. Huybrechts, Lectures on K3 surfaces, Cambridge University Press, Cambridge, U.K. (2016).

[64] M. Schuett and T. Shioda, Elliptic surfaces, Adv. Stud. Pure Math. 60 (2010) 51 [arXiv: 0907.0298]. 
[65] S.H. Katz, A. Klemm and C. Vafa, $M$ theory, topological strings and spinning black holes, Adv. Theor. Math. Phys. 3 (1999) 1445 [hep-th/9910181] [InSPIRE].

[66] C. Lawrie, S. Schäfer-Nameki and T. Weigand, Chiral $2 d$ theories from $N=4 S Y M$ with varying coupling, JHEP 04 (2017) 111 [arXiv: 1612.05640] [INSPIRE].

[67] M. Bershadsky, A. Johansen, V. Sadov and C. Vafa, Topological reduction of $4 D S Y M$ to $2 D$ sigma models, Nucl. Phys. B 448 (1995) 166 [hep-th/9501096] [INSPIRE].

[68] P.S. Aspinwall and R. Kallosh, Fixing all moduli for M-theory on K3 $\times$ K3, JHEP 10 (2005) 001 [hep-th/0506014] [INSPIRE].

[69] J. Kollar, Deformations of elliptic Calabi-Yau manifolds, arXiv:1206.5721 [INSPIRE].

[70] K. Oguiso, On algebraic fiber space structures on a Calabi-Yau 3-fold, Internat. J. Math. 04 (1993) 439.

[71] P.M.H. Wilson, The existence of elliptic fibre space structures on Calabi-Yau threefolds, Math. Annalen 300 (1994) 693. 